\title{
DEVELOPMENT OF THE SPACE CODE FOR NUCLEAR POWER PLANTS
}

\author{
SANG JUN HA ${ }^{1, *}$, CHAN EOK PARK ${ }^{2}$, KYUNG DOO KIM ${ }^{3}$, and CHANG HWAN BAN ${ }^{4}$ \\ ${ }^{1}$ Korea Electric Power Corporation Research Institute \\ 105 Munji-Ro, Yuseong-Gu, Daejeon 305-760, Korea \\ ${ }^{2} \mathrm{KEPCO}$ Engineering \& Construction Company Inc \\ 150 Deokjin-dong, Yuseong-gu, Daejeon, 305-353, Korea \\ ${ }^{3}$ Korea Atomic Energy Research Institute \\ Daejon, Yusong P.O. Box 105, Korea Chang Hwan Ban \\ ${ }^{4}$ Korea Nuclear Fuel \\ 1047 Daedukdaero, Yuseong-Gu, Daejeon 305-353, Korea \\ "Corresponding author. E-mail : hsj@kepri.re.kr
}

Received July 06, 2010

Accepted for Publication September 27, 2010

The Korean nuclear industry is developing a thermal-hydraulic analysis code for safety analysis of pressurized water reactors (PWRs). The new code is called the Safety and Performance Analysis Code for Nuclear Power Plants (SPACE). The SPACE code adopts advanced physical modeling of two-phase flows, mainly two-fluid three-field models which comprise gas, continuous liquid, and droplet fields and has the capability to simulate 3D effects by the use of structured and/or nonstructured meshes. The programming language for the SPACE code is C++ for object-oriented code architecture. The SPACE code will replace outdated vendor supplied codes and will be used for the safety analysis of operating PWRs and the design of advanced reactors. This paper describes the overall features of the SPACE code and shows the code assessment results for several conceptual and separate effect test problems.

KEYWORDS : System Code, SPACE, Nuclear Safety Analysis, Thermal-Hydraulics, Multi-Fields Model, Code Validation

\section{INTRODUCTION}

The Korean nuclear industry has various types of nuclear power plants in operation and under construction, namely, OPR1000, APR1400, Westinghouse 2- or 3-loop plants, ABB-CE 2-loop plants, Framatome 3-loop plants, and CANDU-type plants. For current safety analysis of nuclear power plants, foreign vendor supplied safety analysis codes have been used. In addition, most vendor analysis codes were developed in 1970s and no major changes have been made since then. Therefore, the Korean nuclear industry launched the SPACE code development project in 2006 to develop a new thermal-hydraulics system analysis code by adopting advanced physical modeling of two-phase flows, mainly using multi-field models and with a multi-dimensional simulation capability by the use of structured and/or non-structured meshes. Also, the programming language for the SPACE code is $\mathrm{C}++$ for the new generation of engineers who are more comfortable with $\mathrm{C} / \mathrm{C}++$ than FORTRAN language. The SPACE code will replace outdated vendor supplied codes and will be used for the safety analysis of operating PWRs and the design of advanced water reactors.

The SPACE code is composed of input and output packages, a hydrodynamic model package, a heat structure model, a control system model, a reactor kinetic model, and so on. The hydrodynamic model package is composed of a hydraulic solver, constitutive models, special process models, and component models. The input/output package performs read of input file and restart file, input error check, storage space setup, as well as variable initialization, preparation of main output file, plot files, and restart file. The hydraulic solver adopts two-fluid, three-field governing equations which are comprised of gas, continuous liquid, and droplet fields. The three fields are allowed to be at non-homogeneous and non-equilibrium state, while the gas field is assumed to be a homogenous equilibrium mixture of vapor and non-condensable gas. The governing equations also involve porosity to take into account the structural material impact on the fluid flow [1]. Each field equation is discretized by applying the finite volume method (FVM) [2, 3] to the very unique SPACE mesh 
system which naturally encompasses various threedimensional structured and or non-structured mesh systems, as well as one-dimensional pipe meshes [4]. Non-linear terms appearing in the temporal and source terms of the phasic mass and energy equations are linearized by using the Taylor expansion technique. A semi-implicit scheme [5] is chosen as the basic numerical time advancement scheme in SPACE.

The proper physical models can significantly improve the accuracy of the prediction of a nuclear reactor system's behavior under many different transient conditions because those models are composed of the source terms for the governing equations. To develop the physical models and correlations for the SPACE code, various models currently used in major nuclear reactor system analysis codes have been reviewed, including RELAP5 [6], TRAC-M [7], COBRA-TF [8] , CATHARE [9] and MARS [10]. In addition, a literature survey of recent studies has been performed to incorporate up-to-date models into the SPACE code. Unlike RELAP5, TRACE, and CATHARE, which are the major best-estimate nuclear reactor system analysis codes that only consider liquid and vapor phases, the SPACE code incorporates a dispersed liquid field in addition to vapor and continuous liquid fields. Interfacial interaction models between continuous, dispersed liquid phases, and vapor phase have to be developed separately. Constitutive models of the SPACE comprise the surface area and surface heat transmission correlation, surfacewall friction correlation, droplet separation and adhesion correlations, wall-fluid heat transmission mode and correlations, all correlations required for governing equations, and the type of the correlation is determined by the flow-form map. The SPACE code contains special process and system component models to limit or modify the solution of the basic governing equations reflecting the physical phenomena and to provide the capability to simulate the systems of nuclear power plants.

Although the present SPACE code was designed to simulate nuclear reactor system transients, its application can be expanded to the non-nuclear field including phases of water, vapor, and non-condensable gas.

This paper describes the overall features of the SPACE code, including hydraulic models and physical models, and shows the code assessment results for several conceptual and separate effect test problems.

\section{MODELS OF SPACE CODE}

\subsection{Hydraulic Solver}

\subsubsection{Governing Equations}

The SPACE two-fluid, three field formulation uses a separate set of conservation equations for each phase. The effects of one phase on another are accounted for by interaction terms appearing in the equations. Dividing the liquid phase into two fields is a convenient and physically reasonable way of handling flows where the liquid can appear in both film and droplet form, because the thermal and hydraulic behavior of droplets can be quite different from that of film. The mass conservation equations of vapor, continuous liquid, droplet phase, and noncondensable gas are given as follows:

Continuity equation for vapor phase:

$$
\varepsilon \frac{\partial}{\partial t}\left(\alpha_{g} \rho_{v}\right)+\nabla \cdot\left(\varepsilon \alpha_{g} \rho_{v} \mathbf{U}_{g}\right)=\varepsilon\left(\Gamma_{l}+\Gamma_{d}\right)
$$

Continuity equation for continuous liquid phase:

$$
\varepsilon \frac{\partial}{\partial t}\left(\alpha_{l} \rho_{l}\right)+\nabla \cdot\left(\varepsilon \alpha_{l} \rho_{l} \mathbf{U}_{l}\right)=\varepsilon\left(-\Gamma_{l}-S_{E}+S_{D}\right)
$$

Continuity equation for droplet phase:

$$
\varepsilon \frac{\partial}{\partial t}\left(\alpha_{d} \rho_{d}\right)+\nabla \cdot\left(\varepsilon \alpha_{d} \rho_{d} \mathbf{U}_{d}\right)=\varepsilon\left(-\Gamma_{d}+S_{E}-S_{D}\right)
$$

Continuity equation for non-condensable gas:

$$
\varepsilon \frac{\partial}{\partial t}\left(\alpha_{g} \rho_{n}\right)+\nabla \cdot\left(\varepsilon \alpha_{g} \rho_{n} \mathbf{U}_{g}\right)=0
$$

In the above conservation equations, the subscripts $\mathrm{v}$, $\ell, d, g$, and $\mathrm{n}$ refer to the vapor, continuous liquid, droplet, vapor/non-condensable gas mixture, and noncondensable gas mixture, respectively. The mass transfer due to phase change, denoted by $\Gamma_{l}, \Gamma_{d}$ occurs at continuous liquid-vapor and droplet-vapor interfaces. Also, the two liquid fields can exchange mass by entrainment or de-entrainment, which are denoted by $S_{E}, S_{D}$ respectively.

The momentum conservation equations of gas, continuous liquid, and droplet phase are given as follows:

Momentum equation for vapor phase:

$$
\begin{aligned}
\varepsilon & \frac{\partial \mathbf{U}_{g}}{\partial t}+\nabla \cdot\left(\varepsilon \mathbf{U}_{g} \mathbf{U}_{g}\right)-\mathbf{U}_{g} \nabla \cdot\left(\varepsilon \mathbf{U}_{g}\right) \\
= & -\frac{\varepsilon}{\rho_{g}} \nabla P-\frac{\varepsilon F_{w g}}{\alpha_{g} \rho_{g}} \mathbf{U}_{g}-\frac{\varepsilon F_{g d}}{\alpha_{g} \rho_{g}}\left(\mathbf{U}_{g}-\mathbf{U}_{d}\right)-\frac{\varepsilon F_{g l}}{\alpha_{g} \rho_{g}}\left(\mathbf{U}_{g}-\mathbf{U}_{l}\right)+\varepsilon \mathbf{B} \\
& +\frac{\varepsilon}{\alpha_{g} \rho_{g}}\left(\Gamma_{l, E} \mathbf{U}_{l}+\Gamma_{d, E} \mathbf{U}_{d}-\Gamma_{l, E} \mathbf{U}_{g}-\Gamma_{d, E} \mathbf{U}_{g}\right) \\
& -\varepsilon C_{g, g d} \alpha_{d} \frac{\rho_{m, g d}}{\rho_{g}} \frac{\partial\left(\mathbf{U}_{g}-\mathbf{U}_{d}\right)}{\partial t}-\varepsilon C_{g, g l} \alpha_{l} \frac{\rho_{m, g l}}{\rho_{g}} \frac{\partial\left(\mathbf{U}_{g}-\mathbf{U}_{l}\right)}{\partial t}
\end{aligned}
$$

Momentum equation for continuous liquid phase:

$$
\begin{aligned}
& \varepsilon \frac{\partial \mathbf{U}_{l}}{\partial t}+\nabla \cdot\left(\varepsilon \mathbf{U}_{l} \mathbf{U}_{l}\right)-\mathbf{U}_{l} \nabla \cdot\left(\varepsilon \mathbf{U}_{l}\right) \\
= & -\frac{\varepsilon}{\rho_{l}} \nabla P-\frac{\varepsilon F_{w l}}{\alpha_{l} \rho_{l}} \mathbf{U}_{l}-\frac{\varepsilon F_{l g}}{\alpha_{l} \rho_{l}}\left(\mathbf{U}_{l}-\mathbf{U}_{g}\right)+\varepsilon \mathbf{B} \\
& +\frac{\varepsilon}{\alpha_{l} \rho_{l}}\left(-\Gamma_{l, C} \mathbf{U}_{l}+\Gamma_{l, C} \mathbf{U}_{g}+S_{D} \mathbf{U}_{d}-\mathbf{U}_{l} S_{D}\right)-\varepsilon C_{g, g l} \alpha_{g} \frac{\rho_{m, g l}}{\rho_{l}} \frac{\partial\left(\mathbf{U}_{l}-\mathbf{U}_{g}\right)}{\partial t},
\end{aligned}
$$


where $\Gamma_{l}=\Gamma_{l, E}-\Gamma_{l, C}$.

Momentum equation for droplet phase:

$\varepsilon \frac{\partial \mathbf{U}_{d}}{\partial t}+\nabla \cdot\left(\varepsilon \mathbf{U}_{d} \mathbf{U}_{d}\right)-\mathbf{U}_{d} \nabla \cdot\left(\varepsilon \mathbf{U}_{d}\right)$
$=-\frac{\varepsilon}{\rho_{d}} \nabla P-\frac{\varepsilon F_{w d}}{\alpha_{d} \rho_{d}} \mathbf{U}_{d}-\frac{\varepsilon F_{d g}}{\alpha_{d} \rho_{d}}\left(\mathbf{U}_{d}-\mathbf{U}_{g}\right)+\varepsilon \mathbf{B}$
$+\frac{\varepsilon}{\alpha_{d} \rho_{d}}\left(-\Gamma_{d, C} \mathbf{U}_{d}+\Gamma_{d, C} \mathbf{U}_{g}+S_{E} \mathbf{U}_{l}-S_{E} \mathbf{U}_{d}\right)-\varepsilon C_{g, d g} \alpha_{g} \frac{\rho_{m, d g}}{\rho_{d}} \frac{\partial\left(\mathbf{U}_{d}-\mathbf{U}_{g}\right)}{\partial t}$,

where $\Gamma_{d}=\Gamma_{d, E}-\Gamma_{d, C}$.

The energy conservation equation is set up for each of the vapor/gas mixture, continuous liquid, and droplet phases, under the assumption that the three phases are at a thermal non-equilibrium state.

$$
\begin{gathered}
\frac{\partial\left(\varepsilon \alpha_{g}\left(\rho_{v} e_{v}+\rho_{n} e_{n}\right)\right)}{\partial t}+\nabla \cdot\left(\varepsilon \alpha_{v}\left(\rho_{v} e_{v}+\rho_{n} e_{n}\right) \mathbf{U}_{g}\right) \\
=-\varepsilon P \frac{\partial \alpha_{g}}{\partial t}-P \nabla \cdot\left(\varepsilon \alpha_{g} \mathbf{U}_{g}\right) \\
+\varepsilon\left(Q_{i v-l}+\Gamma_{l} h_{v l}^{*}+\Gamma_{d} h_{v d}^{*}+Q_{i v-d}+Q_{l-n}+Q_{d-n}\right) \\
\frac{\partial\left(\varepsilon \alpha_{l} \rho_{l} e_{l}\right)}{\partial t}+\nabla \cdot\left(\varepsilon \alpha_{l} \rho_{l} e_{l} \mathbf{U}_{l}\right)= \\
+\varepsilon P \frac{\partial \alpha_{l}}{\partial t}-P \nabla \cdot\left(\varepsilon \alpha_{l} \mathbf{U}_{l}\right) \\
\left.+Q_{i l}-\Gamma_{l} h_{l}^{*}-S_{E} h_{l}+S_{D} h_{d}-Q_{l-n}\right) \\
\frac{\partial\left(\varepsilon \alpha_{d} \rho_{d} e_{d}\right)}{\partial t}+\nabla \cdot\left(\varepsilon \alpha_{d} \rho_{d} e_{d} \mathbf{U}_{d}\right)= \\
+\varepsilon P \frac{\partial \alpha_{d}}{\partial t}-P \nabla \cdot\left(\varepsilon \alpha_{d} \mathbf{U}_{d}\right) \\
+\varepsilon\left(Q_{i d}-\Gamma_{d} h_{d}^{*}+S_{E} h_{l}-S_{D} h_{d}-Q_{d-n}\right)
\end{gathered}
$$

In the above phasic energy conservation equations, the interfacial heat transfer rate per volume is denoted by $\mathrm{Q}$.

\subsubsection{Mesh System}

The staggered mesh system of SPACE is based on the orthogonal hexahedral shape of a cell and its surrounding faces. All of the geometric quantities are described in terms of cell volume, centroid, face area, and face center, so that Cartesian and cylindrical mesh systems can be expressed in the same manner. Each scalar cell normally has six faces in three-dimensional Cartesian or cylindrical mesh blocks. However, two-dimensional Cartesian meshes or one-dimensional pipe can be also represented only by reducing the numbers of the surrounding faces. The direction of a cell and the associated faces can be distinguished by the unique number sequentially given to each face. Each momentum cell is shifted by the half size of a scalar cell so that it consists of the front half of the owner scalar cell and the back half of the neighbor scalar cell. Each face can be divided into several sub-faces. With these subfaces, one-dimensional or three-dimensional branches are easily modeled in the SPACE code staggered mesh system. Generally curved pipes can also be represented by providing an inclined angle to each scalar cell.

\subsubsection{Discretization and Linearization}

Using the divergence theorem, the mass and energy equations can be expressed as follows:

Non-condensable gas mass conservation eguation:

$$
\varepsilon V_{P} \frac{\alpha_{g}^{n} \rho_{n}^{n}-\alpha_{g} \rho_{n}}{\Delta t}+\sum_{E \in P} \varepsilon^{E d} \alpha_{g}^{E d} \rho_{n}^{E}\left(t_{P}^{E} U_{g n}^{n} A^{E}\right)=0
$$

Vapor mass conservation equation:

$$
\varepsilon V_{P} \frac{\alpha_{g}^{n} \rho_{v}^{n}-\alpha_{g} \rho_{v}}{\Delta t}+\sum_{E \in P} \varepsilon^{E d} \alpha_{g}^{E d} \rho_{v}^{E}\left(t_{P}^{E} U_{g n}^{n} A^{E}\right)=\varepsilon V_{P}\left(\Gamma_{l}+\Gamma_{d}\right)^{n}
$$

Droplet mass conservation equation:

$$
\begin{aligned}
\varepsilon V_{P} \frac{\alpha_{d}^{n} \rho_{d}^{n}-\alpha_{d} \rho_{d}}{\Delta t} & +\sum_{E \in P} \varepsilon^{E d} \alpha_{d}^{E d} \rho_{d}^{E}\left(l_{P}^{E} U_{d n}^{n} A^{E}\right) \\
& =\varepsilon V_{P}\left(-\Gamma_{d}^{n}+S_{E}-S_{D}\right)
\end{aligned}
$$

Continuous liquid mass conservation equation:

$$
\begin{aligned}
\varepsilon V_{P} \frac{\alpha_{l}^{n} \rho_{l}^{n}-\alpha_{l} \rho_{l}}{\Delta t} & +\sum_{E \in P} \varepsilon^{E d} \alpha_{l}^{E d} \rho_{l}^{E}\left(l_{P}^{E} U_{l n}^{n} A^{E}\right) \\
& =\varepsilon V_{P}\left(-\Gamma_{l}^{n}-S_{E}+S_{D}\right)
\end{aligned}
$$

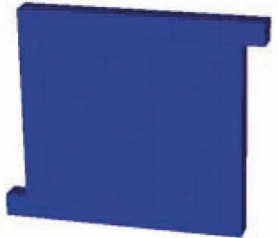

(a) Cartesian mesh

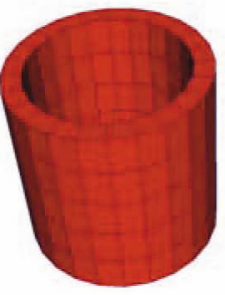

(b) cylindrical mesh

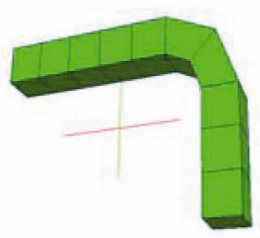

(c) curved pipe mesh

Fig. 1. Examples of Mesh Type Represented by the SPACE Staggered Mesh System 
Gas energy conservation equation:

$\varepsilon V_{P} \frac{\alpha_{g}^{n}\left(\rho_{v}^{n} e_{v}^{n}+\rho_{n}^{n} e_{n}^{n}\right)-\alpha_{g}\left(\rho_{v} e_{v}+\rho_{n} e_{n}\right)}{\Delta t}$

$+\sum_{E \in P} \boldsymbol{t}_{P}^{E} \varepsilon^{E d} \alpha_{g}^{E}\left({ }^{d} \rho_{v}^{E d} e_{v}^{E}+{ }^{d} \rho_{n}^{E d} e_{n}^{E}\right)\left(U_{g n}^{n} A^{E}\right)+P \sum_{E \in P} \boldsymbol{t}_{P}^{E} \varepsilon^{E d} \alpha_{g}^{E} U_{g n}^{n} A^{E}$

$=\varepsilon V_{P}\left[-P \frac{\alpha_{g}^{n}-\alpha_{g}}{\Delta t}+Q_{i v-l}^{n}+\Gamma_{l}^{n} h_{v l}^{*}+\Gamma_{d}^{n} h_{v d}^{*}+Q_{i v-d}^{n}+Q_{l-n}^{n}+Q_{d-n}^{n}+Q_{w g}\right]$

Droplet energy conservation equation:

$\varepsilon V_{P} \frac{\alpha_{d}^{n} \rho_{d}^{n} e_{d}^{n}-\alpha_{d} \rho_{d} e_{d}}{\Delta t}$

$+\sum_{E \in P} t_{P}^{E} \varepsilon^{E d} \alpha_{d}^{E d} \rho_{d}^{E d} e_{d}^{E}\left(U_{d n}^{n} A^{E}\right)+P \sum_{E \in P} t_{P}^{E} \varepsilon^{E d} \alpha_{d}^{E} U_{d n}^{n} A^{E}$

$=\varepsilon V_{P}\left[-P \frac{\alpha_{d}^{n}-\alpha_{d}}{\Delta t}+Q_{i d}^{n}-\Gamma_{d}^{n} h_{d}^{*}+S_{E} h_{l}-S_{D} h_{d}-Q_{d-n}^{n}+Q_{w d}\right]$

Continuous liquid energy conservation equation:

$$
\begin{aligned}
& \varepsilon V_{P} \frac{\alpha_{l}^{n} \rho_{l}^{n} e_{l}^{n}-\alpha_{l} \rho_{l} e_{l}}{\Delta t} \\
& +\sum_{E \in P} \iota_{P}^{E} \varepsilon^{E d} \alpha_{l}^{E d} \rho_{l}^{E d} e_{l}^{E}\left(U_{l n}^{n} A^{E}\right)+P \sum_{E \in P} t_{P}^{E} \varepsilon^{E d} \alpha_{l}^{E} U_{l n}^{n} A^{E} \\
& =\varepsilon V_{P}\left[-P \frac{\alpha_{l}^{n}-\alpha_{l}}{\Delta t}+Q_{i l}^{n}-\Gamma_{l}^{n} h_{l}^{*}-S_{E} h_{l}+S_{D} h_{d}-Q_{l-n}^{n}+Q_{w l}\right]
\end{aligned}
$$

In the hexahedral shape of a scalar cell, each orthogonal face can be categorized into three different types, depending on the direction of the face vector. The components of the cell velocity vector, $\mathrm{U}_{(\mathrm{k})}$, are calculated by averaging the same type face velocities. If the transverse direction velocity is denoted by $\mathrm{XU}_{(\mathrm{k})}$ at each face, the momentum equations can be expressed as follows:

Gas momentum conservation equation:

$\varepsilon^{E} V^{E} \frac{\left(U_{g n}^{E}\right)^{n}-U_{g n}^{E}}{\Delta t}+\varepsilon_{(k)}^{\text {Neighbor d }} U_{g(k)}^{\text {Neighbor }}\left(A_{(k)}^{\text {Neighbor }} U_{g(k)}^{\text {Neighbor }}\right)$

$-\varepsilon_{(k)}^{\text {Owner } d} U_{g(k)}^{O \text { wner }}\left(A_{(k)}^{O_{\text {wner }}} U_{g(k)}^{\text {Owner }}\right)+\sum_{E^{\prime} \in \text { Owner }, \text { trp } \neq k} \varepsilon^{E^{\prime} d} X U_{g(k)}^{\text {Fhcell }}\left(l_{\text {Owner }}^{E^{\prime}} \frac{1}{2} U_{g n}^{E^{\prime}} A^{E^{\prime}}\right)$

$+\sum_{E^{\prime} \in \text { Neighbor,typ } \neq k} \varepsilon^{E^{\prime} d} X U_{g(k)}^{\text {Bhcell }}\left(l_{\text {Neighbor }}^{E^{\prime}} \frac{1}{2} U_{g n}^{E^{\prime}} A^{E^{\prime}}\right)$

$+F_{g(k)} V^{E}-U_{g n}^{E} \varepsilon_{(k)}^{\text {Neighbor }}\left(A_{(k)}^{\text {Neighbor }} U_{g(k)}^{\text {Neighbor }}\right)+U_{g n}^{E} \varepsilon_{(k)}^{\text {Owner }}\left(A_{(k)}^{\text {Owner }} U_{g(k)}^{\text {Owner }}\right)$

$-U_{g n}^{E} \sum_{E^{\prime} \in O \text { Wner, }, \text { yp } \neq k} \varepsilon^{E^{\prime}}\left(l_{\text {Owner }}^{E^{\prime}} \frac{1}{2} U_{g n}^{E^{\prime}} A^{E^{\prime}}\right)-U_{g n}^{E} \sum_{E^{\prime} \in \text { Neighbor, typ } \neq k} \varepsilon^{E^{\prime}}\left(l_{\text {Neighbor }}^{E^{\prime}} \frac{1}{2} U_{g n}^{E^{\prime}} A^{E^{\prime}}\right)$

$=\frac{1}{\rho_{g}} \varepsilon^{E} A^{E}\left(P_{\text {Owner }}^{n}-P_{\text {Neighbor }}^{n}\right)$

$$
+\frac{\varepsilon^{E} V^{E}}{\alpha_{g} \rho_{g}}\left[\begin{array}{l}
-F_{w g}\left(U_{g n}^{E}\right)^{n}-F_{g d}\left(U_{g n}^{E}-U_{d n}^{E}\right)^{n}-F_{g l}\left(U_{g n}^{E}-U_{l n}^{E}\right)^{n}+\alpha_{g} \rho_{g} \mathbf{B} \cdot \mathbf{n}^{E} \\
+\Gamma_{l, E}\left(U_{l n}^{E}\right)^{n}+\Gamma_{d, E}\left(U_{d n}^{E}\right)^{n}-\Gamma_{l, E}\left(U_{g n}^{E}\right)^{n}-\Gamma_{d, E}\left(U_{g n}^{E}\right)^{n} \\
-C_{g, g d} \alpha_{g} \alpha_{d} \rho_{m, g d} \frac{\left(U_{g n}^{E}-U_{d n}^{E}\right)^{n}-\left(U_{g n}^{E}-U_{d n}^{E}\right)}{\Delta t} \\
-C_{g, g l} \alpha_{g} \alpha_{l} \rho_{m, g l} \frac{\left(U_{g n}^{E}-U_{l n}^{E}\right)^{n}-\left(U_{g n}^{E}-U_{l n}^{E}\right)}{\Delta t}
\end{array}\right]
$$

Droplet momentum conservation equation:

$$
\begin{aligned}
& \varepsilon^{E} V^{E} \frac{\left(U_{d n}^{E}\right)^{n}-U_{d n}^{E}}{\Delta t}+\varepsilon_{(k)}^{\text {Neighbor d }} U_{d(k)}^{\text {Neighbor }}\left(A_{(k)}^{\text {Neighbor }} U_{d(k)}^{\text {Neighbor }}\right) \\
& -\varepsilon_{(k)}^{\text {Owner } d} U_{d(k)}^{\text {Owner }}\left(A_{(k)}^{\text {Owner }} U_{d(k)}^{\text {Owner }}\right)+\sum_{E^{\prime} \in O_{\text {wner }, t y p \neq k}} \varepsilon^{E^{\prime} d} X U_{d(k)}^{\text {Fhcell }}\left(i_{\text {Owner }}^{E^{\prime}} \frac{1}{2} U_{d n}^{E^{\prime}} A^{E^{\prime}}\right) \\
& +\sum_{E^{\prime} \in \text { Neighbor, }, y p \neq k} \varepsilon^{E^{\prime} d} X U_{d(k)}^{\text {Bhcell }}\left(l_{\text {Neighbor }}^{E^{\prime}} \frac{1}{2} U_{d n}^{E^{\prime}} A^{E^{\prime}}\right) \\
& +F_{d(k)} V^{E}-U_{d n}^{E} \varepsilon_{(k)}^{\text {Neighbor }}\left(A_{(k)}^{\text {Neighbor }} U_{d(k)}^{\text {Neighbor }}\right)+U_{d n}^{E} \varepsilon_{(k)}^{\text {Owner }}\left(A_{(k)}^{\text {Owner }} U_{d(k)}^{\text {Owner }}\right) \\
& -U_{d n}^{E} \sum_{E^{\prime} \in O \text { wner }, \text { typ } \neq k} \varepsilon^{E^{\prime}}\left(l_{\text {owner }}^{E^{\prime}} \frac{1}{2} U_{d n}^{E^{\prime}} A^{E^{\prime}}\right)-U_{d n}^{E^{\prime}} \sum_{E \in \text { Neighbor, typ } \neq k} \varepsilon^{E^{\prime}}\left(l_{\text {Neighbor }}^{E^{\prime}} \frac{1}{2} U_{d n}^{E^{\prime}} A^{E^{\prime}}\right) \\
& =\frac{1}{\rho_{d}} \varepsilon^{E} A^{E}\left(P_{\text {Owner }}^{n}-P_{\text {Neighbor }}^{n}\right) \\
& +\frac{\varepsilon^{E} V^{E}}{\alpha_{d} \rho_{d}}\left[\begin{array}{l}
-F_{w d}\left(U_{d n}^{E}\right)^{n}-F_{d \mathrm{~g}}\left(U_{d n}^{E}-U_{g n}^{E}\right)^{n}+\alpha_{d} \rho_{d} \mathbf{B} \cdot \mathbf{n}^{E} \\
-\Gamma_{d, C}\left(U_{d n}^{E}\right)^{n}+\Gamma_{d, C}\left(U_{g n}^{E}\right)^{n}+S_{E}\left(U_{l n}^{E}\right)^{n}-S_{E}\left(U_{d n}^{E}\right)^{n} \\
-C_{g, d \mathrm{~g}} \alpha_{d} \alpha_{g} \rho_{m, d \mathrm{~g}} \frac{\left(U_{d n}^{E}-U_{g n}^{E}\right)^{n}-\left(U_{d n}^{E}-U_{g n}^{E}\right)}{\Delta t}
\end{array}\right]
\end{aligned}
$$

Continuous liquid momentum conservation equation:

$$
\begin{aligned}
& \varepsilon^{E} V^{E} \frac{\left(U_{l n}^{E}\right)^{n}-U_{l n}^{E}}{\Delta t}+\varepsilon_{(k)}^{\text {Neighbor } d} U_{l(k)}^{\text {Neighbor }}\left(A_{(k)}^{\text {Neighbor }} U_{l(k)}^{\text {Neighbor }}\right) \\
& -\varepsilon_{(k)}^{\text {Owner } d} U_{l(k)}^{\text {Owner }}\left(A_{(k)}^{\text {Owner }} U_{l(k)}^{\text {Owner }}\right)+\sum_{E^{\prime} \in O \text { wner, }, \text { typ } \neq k} \varepsilon^{E^{\prime} d} X U_{l(k)}^{\text {Fhcell }}\left(l_{\text {Owner }}^{E^{\prime}} \frac{1}{2} U_{l n}^{E^{\prime}} A^{E^{\prime}}\right) \\
& +\sum_{E^{\prime} \in \text { Neighbor,typ } \neq k} \varepsilon^{E^{\prime} d} X U_{l(k)}^{\text {Bhcell }}\left(u_{\text {Neighbor }}^{E^{\prime}} \frac{1}{2} U_{l n}^{E^{\prime}} A^{E^{\prime}}\right) \\
& +F_{l(k)} V^{E}-U_{l n}^{E} \varepsilon_{(k)}^{\text {Neighbor }}\left(A_{(k)}^{\text {Neighbor }} U_{l(k)}^{\text {Neighbor }}\right)+U_{l n}^{E} \varepsilon_{(k)}^{\text {Owner }}\left(A_{(k)}^{\text {Owner }} U_{l(k)}^{\text {Owner }}\right) \\
& -U_{l n}^{E} \sum_{E^{\prime} \in O \text { wner }, \text { typ } \neq k} \varepsilon^{E^{\prime}}\left(l_{\text {lwner }}^{E^{\prime}} \frac{1}{2} U_{l n}^{E^{\prime}} A^{E^{\prime}}\right)-U_{l n}^{E} \sum_{E^{\prime} \in \text { Neighbor, }, \text { typ } \neq k} \varepsilon^{E^{\prime}}\left(l_{\text {Neighbor }}^{E^{\prime}} \frac{1}{2} U_{l n}^{E^{\prime}} A^{E^{\prime}}\right) \\
& =\frac{1}{\rho_{l}} \varepsilon^{E} A^{E}\left(P_{\text {Owner }}^{n}-P_{\text {Neighbor }}^{n}\right) \\
& +\frac{\varepsilon^{E} V^{E}}{\alpha_{l} \rho_{l}}\left[\begin{array}{l}
-F_{w l}\left(U_{l n}^{E}\right)^{n}-F_{\mathrm{lg}}\left(U_{l n}^{E}-U_{g n}^{E}\right)^{n}+\alpha_{l} \rho_{l} \mathbf{B} \cdot \mathbf{n}^{E} \\
-\Gamma_{l, C}\left(U_{l n}^{E}\right)^{n}+\Gamma_{l, C}\left(U_{g n}^{E}\right)^{n}+S_{D}\left(U_{d n}^{E}\right)^{n}-S_{D}\left(U_{l n}^{E}\right)^{n} \\
-C_{g, l g} \alpha_{l} \alpha_{g} \rho_{m, l g} \frac{\left(U_{l n}^{E}-U_{g n}^{E}\right)^{n}-\left(U_{l n}^{E}-U_{g n}^{E}\right)}{\Delta t}
\end{array}\right]
\end{aligned}
$$

The additional force terms, $\mathrm{F}_{\phi(k)}$, representing the centrifugal and Coriolis force are given as

$$
\begin{aligned}
& \text { Cartesian coordinate }\left(x, y, z: U_{x}, U_{y}, U_{z}\right): \\
& F_{\phi(1)}=0, \quad F_{\phi(2)}=0, \quad F_{\phi(3)}=0 \\
& \text { Cylindrical coordinate }\left(r, \theta, z: U_{r}, U_{\theta}, U_{z}\right): \\
& F_{\phi(1)}=-\frac{U_{\phi(2)}^{2}}{r}, \quad F_{\phi(2)}=\frac{U_{\phi(1)} U_{\phi(2)}}{r}, \quad F_{\phi(3)}=0
\end{aligned}
$$

When a Newton-Raphson type of solver is used, the non-linear terms need to be linearized with respect to the primitive variables. The primitive variables used in SPACE are partial pressure of the non-condensable gas mixture 
$\left(\mathrm{P}_{\mathrm{n}}\right)$, phasic temperatures $\left(T_{g}, T_{i}, T_{d}\right)$, phasic volume fractions $\left(\alpha_{v}, \alpha_{d}\right)$ total pressure $(\mathrm{P})$, and phasic velocities $\left(\mathbf{U}_{g}, \mathbf{U}_{i}, \mathbf{U}_{d},\right)$. For the purpose of linearization, the nonprimitive variables appearing in the temporal terms, such as density and energy, are expanded with respect to the primitive variables, applying the first-order Taylor expansion technique:

$$
\begin{gathered}
\delta \rho_{v}=\left(\frac{\partial \rho_{v}}{\partial P}\right) \delta P+\left(\frac{\partial \rho_{v}}{\partial P_{n}}\right) \delta P_{n}+\left(\frac{\partial \rho_{v}}{\partial T_{g}}\right) \delta T_{g} \\
\delta \rho_{l}=\left(\frac{\partial \rho_{l}}{\partial P}\right) \delta P+\left(\frac{\partial \rho_{l}}{\partial T_{l}}\right) \delta T_{l} .
\end{gathered}
$$

Since phase change terms appearing in the source of phasic energy equations are functions of saturation temperature, the saturation temperature also needs to be expanded with respect to the primitive variables for the source term linearization:

$$
\delta T^{s}=\left(\frac{\partial T^{s}}{\partial P}\right) \delta P+\left(\frac{\partial T^{s}}{\partial P_{n}}\right) \delta P_{n},
$$

where

$$
\begin{gathered}
\left(\frac{\partial T^{s}}{\partial P}\right)=\left(\frac{\partial T^{S}}{\partial P_{v}}\right)=T_{s} \frac{v_{v}^{s}-v_{l}^{s}}{h_{v}^{s}-h_{l}^{s}} \\
\left(\frac{\partial T^{s}}{\partial P_{n}}\right)=-\left(\frac{\partial T^{S}}{\partial P_{v}}\right)=-T_{s} \frac{v_{v}^{s}-v_{l}^{s}}{h_{v}^{s}-h_{l}^{s}} .
\end{gathered}
$$

\subsubsection{Time Advancement Scheme}

During the discretization of the differential equations, time integration is performed either implicitly or explicitly, depending on the numerical solution scheme. In the semi-implicit scheme, implicitness is selected such that the field equations can be reduced to a single algebraic pressure equation per fluid control volume or mesh cell. The convection terms of momentum equations are discretized in an explicit manner, while the pressure gradient terms are implicitly treated. Then, the semiimplicit face-momentum equations can be expressed as

$$
\left[\begin{array}{lll}
m 11 & m 12 & m 13 \\
m 21 & m 22 & m 23 \\
m 31 & m 32 & m 33
\end{array}\right]\left[\begin{array}{l}
\left(U_{g n}^{E}\right)^{n} \\
\left(U_{l n}^{E}\right)^{n} \\
\left(U_{d n}^{E}\right)^{n}
\end{array}\right]=\left[\begin{array}{l}
S_{g} \\
S_{l} \\
S_{d}
\end{array}\right]+\left[\begin{array}{c}
\frac{\varepsilon^{E} A^{E}}{\rho_{g}} \\
\frac{\varepsilon^{E} A^{E}}{\rho_{l}} \\
\frac{\varepsilon^{E} A^{E}}{\rho_{d}}
\end{array}\right]\left(\begin{array}{l}
\left.P_{\text {Owner }}^{n}-P_{\text {Neighbor }}^{n}\right) \\
\text { (27) }
\end{array}\right]
$$

In the above equation, the matrix coefficoents, $m_{i j}$, and the source vector, $S_{\phi}$, can be obtained directly by rearranging the discretized phasic momentum equations,
(18) to (20), into a matrix form. By inverting the momentum matrix, the phasic velocities are expressed again as a linear function of pressure gradient:

$$
\begin{aligned}
& \left(U_{g n}^{E}\right)^{n}={ }^{e x p} U_{g n}^{E}+\xi_{g}^{E}\left(P_{\text {Owner }}^{n}-P_{\text {Neighbor }}^{n}\right) \\
& \left(U_{l n}^{E}\right)^{n}={ }^{\exp } U_{l n}^{E}+\xi_{l}^{E}\left(P_{\text {Owner }}^{n}-P_{\text {Neighbor }}^{n}\right) \\
& \left(U_{d n}^{E}\right)^{n}={ }^{\exp } U_{d n}^{E}+\xi_{d}^{E}\left(P_{\text {Owner }}^{n}-P_{\text {Neighbor }}^{n}\right) .
\end{aligned}
$$

When using the staggered mesh, the phasic velocities are expressed as a function of the pressure gradient across the momentum cell, $\Delta \mathrm{P}$, which is determined by the adjacent scalar cell pressures.

Once the explicit face-normal velocity and pressure coefficients have been obtained from the momentum equations, the mass and energy equation at a cell can be derived in the following matrix form by substituting the linear relationship between face-normal velocity and the owner-to-neighbor pressure difference into their implicit velocity terms:

$$
\begin{aligned}
{[A][\delta x]=} & {[b g]+\sum_{E \in P}[(P f+g) \xi]_{P}^{E} \delta P } \\
& -\sum_{E \in P}[(P f+g) \xi]_{N}^{E} \delta P_{N},
\end{aligned}
$$

where

$$
\begin{aligned}
& {[b g]=[b]+\sum_{E \in P} t_{P}^{E}[(P f+g) U]^{E},} \\
& {[\delta x]^{T}=\left(\delta P_{n}, \delta T_{g}, \delta T_{d}, \delta T_{l}, \delta \alpha_{g}, \delta \alpha_{d}, \delta P\right) .}
\end{aligned}
$$

In the equation (29), the subscript, $\mathrm{P}$, denotes present cell, and $\mathrm{N}$ represents neighbor cells adjacent to the present cell, whereas in Eq. (27) and (28) "owner" and "neighbor" means the cell from which a face originates, and the one to which the face is directed, respectively. Each component of the cell matrix, $[A]$, and the source vector, $[b g]$, can be derived in a straightforward manner by first applying the linearization technique described in the equations (22) to (24) to the cell mass and energy equations (11) to (17) and then rearranging the linearized equations into a maxtrix form. Each component of the pressure coefficient vector, $[(p f+\mathrm{g}) \xi]^{E}$, is defined as

$$
\left[\begin{array}{l}
{[(P f+g) \xi]_{1}^{E}} \\
{[(P f+g) \xi]_{2}^{E}} \\
{[(P f+g) \xi]_{3}^{E}} \\
{[(P f+g) \xi]_{4}^{E}} \\
{[(P f+g) \xi]_{5}^{E}} \\
{[(P f+g) \xi]_{6}^{E}} \\
{[(P f+g) \xi]_{7}^{E}}
\end{array}\right]:=\left[\begin{array}{l}
\left(P f_{1}^{E}+g_{1}^{E}\right) \xi_{g}^{E} \\
\left(P f_{2}^{E}+g_{2}^{E}\right) \xi_{g}^{E} \\
\left(P f_{3}^{E}+g_{3}^{E}\right) \xi_{d}^{E} \\
\left(P f_{4}^{E}+g_{4}^{E}\right) \xi_{l}^{E} \\
\left(P f_{5}^{E}+g_{5}^{E}\right) \xi_{g}^{E} \\
\left(P f_{6}^{E}+g_{6}^{E}\right) \xi_{d}^{E} \\
\left(P f_{7}^{E}+g_{7}^{E}\right) \xi_{l}^{E}
\end{array}\right],
$$


where

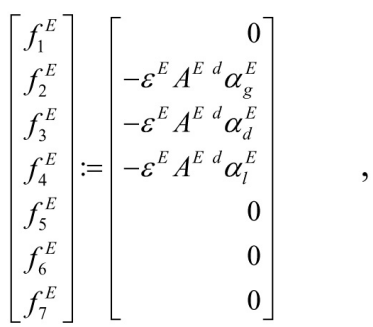

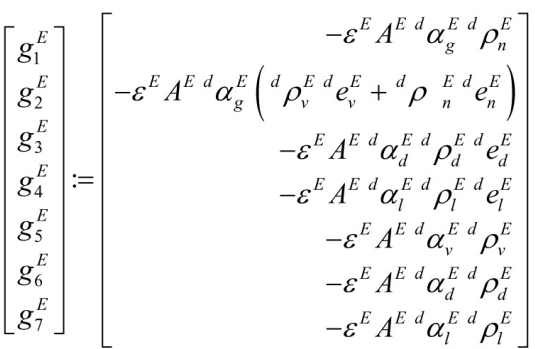

After the cell matrix is constructed, matrix inversion is performed. Then, the solution vector can be written as

$$
\begin{aligned}
{[\delta x]=} & {[A]^{-1}[b g]+[A]^{-1} \sum_{E \in P}[(P f+g) \xi]_{P}^{E} \delta P } \\
& -[A]^{-1} \sum_{E, N \in P}[(P f+g) \xi]_{N}^{E} \delta P_{N}
\end{aligned}
$$

If we let $[a m b g]=[A]^{-1}[b g]$, and $[a m \xi]=[A]^{-1}[(p f+$ $g) \xi]^{E}$ in the equation (34), the last row of cell matrix equations leads to the following pressure equation:

$$
\delta P=a m b g_{7}+\sum_{E \in P} a m \xi_{7}^{E} \delta P-\sum_{E, N \in P} a m \xi_{7}^{E} \delta P_{N} .
$$

Rearranging the pressure equation,

$$
\left(1-\sum_{E \in P} a m \xi_{7}^{E}\right) \delta P+\sum_{E, N \in P} a m \xi_{7 N}^{E} \delta P_{N}=a m b g_{7}
$$

A similar equation may be derived for each cell. This set of equations for the pressure variation in the entire computational cells is called the system pressure matrix. The $\mathrm{N}$ x $\mathrm{N}$ system of pressure equations is solved simultaneously at each time step, using the sparse matrix solver. Once a solution for the linear pressure variation in each cell has been obtained, the linear variation in the other primitive variables is unfolded using Eq. (34). Then, the pressure differences are substituted into Eq. (28) to obtain the new time velocities.

\subsection{Models and Correlations}

The physical models and correlations of the SPACE code are categorized into five packages: i) a flow regime selection package, ii) a wall and interfacial friction package, iii) an interfacial heat and mass transfer package, iv) a droplet entrainment and de-entrainment package, and v) a wall heat transfer package. Detailed descriptions of models and correlation packages for the SPACE code can be found in the models and correlation manual [28].

\subsubsection{Flow Regime Selection Package}

All the interfacial interaction properties, such as interfacial heat and mass transfer rate and interfacial drag force are highly dependent on the flow regime. The SPACE code has two basic flow regime maps: horizontal and vertical flow regime maps. The vertical flow regime map is applied in cells with an inclined sine angle, $2 / 3 \leq \mid \sin$ $\phi \mid \leq 1$, and the horizontal map is applied in cells with inclined sine angle, $0<|\sin \phi| \leq 1 / 3$. Interpolation is used in cells with inclined sine angle, $1 / 3<|\sin \phi| \leq 2 / 3$. Each flow regime map consists of bubbly, slug/capbubble, interpolation, annular-mist, stratified, and transition flow regimes. A horizontal flow regime is determined according to the void fraction and relative velocity (or mass flux for the vertical flow regime map) as shown in Fig. 2. The differences in the flow regime map with other conventional codes are that i) a bubbly flow regime is directly switched to an interpolation regime without passing a slug flow regime if the flow rate is over $2700 \mathrm{~kg} / \mathrm{m}^{2}-\mathrm{s}$, and ii) a mist flow regime does not exist since a droplet fraction is calculated from the hydraulic solver. A hot wall flow regime map has also been incorporated for post-dryout condition.

\subsubsection{Interfacial Area Concentration}

Interfacial area concentration is required to predict the interfacial transport phenomena, such as interfacial heat transfer and interfacial drag force. Two types of interfacial area concentration are calculated for vaporcontinuous liquid interfaces and vapor-dispersed liquid interfaces. Interfacial area concentration for a slug flow is the sum of interfacial area concentrations for small and Taylor bubbles. The interfacial area concentration for annular-mist flow is divided into that of the wall flowing film and core floating droplets. The interfacial area

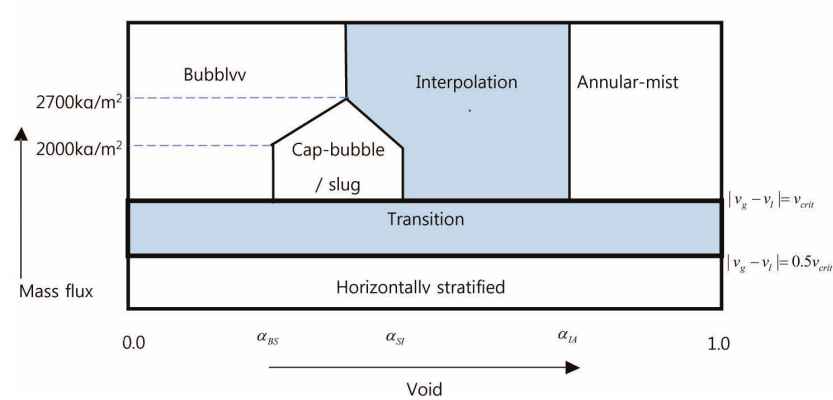

Fig. 2. Horizontal Flow Regime Map 
concentration model developed for each regime [28] has been examined to verify and to make sure of a smooth transition over the flow regime transitions as shown in Fig. 3.

\subsubsection{Interfacial Heat and Mass Transfer Packages}

Interfacial heat transfer is determined by multiplying the temperature difference at an interface with the product of an interfacial area concentration and an interfacial heat transfer coefficient. The interfacial heat transfer coefficient is determined by the degree of superheating or subcooling and the flow regime. For a slug flow, interfacial heat transfer coefficients for small and Taylor bubbles are calculated separately. For small bubbles in a slug flow condition, the same correlation is used as that for bubbly flow. A large constant heat transfer coefficient is applied for physically unstable states, such as subcooled vapor or superheated liquid phase to avoid the sustaining of an unstable state. To ensure a smooth transition and that the coding of the equation is right, the interfacial heat transfer coefficient has been examined by changing the void fraction

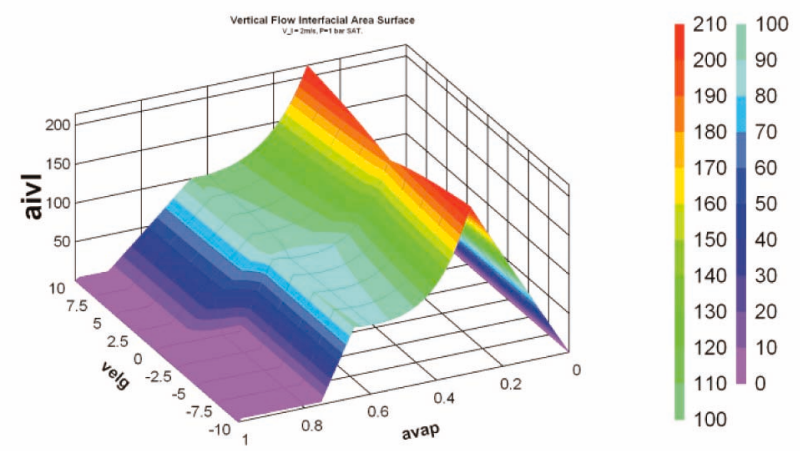

Fig. 3. Interfacial area Concentration as a Function of Vapor Velocity and Void Fraction at Constant Liquid Velocity of 2 $\mathrm{m} / \mathrm{s}$ and Constant Pressure of $0.1 \mathrm{MPa}$ in a Vertical Pipe

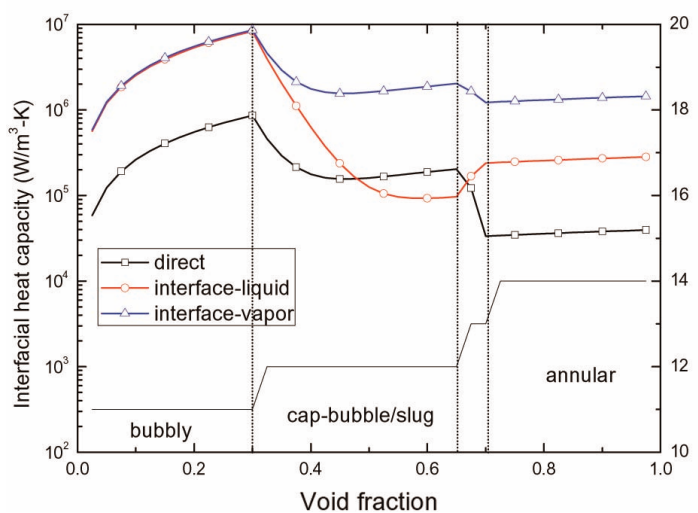

Fig. 4. Interfacial Heat Transfer as a Function of Void Fraction at Constant Phasic Velocities and Constant Pressure of 15 $\mathrm{MPa}$ in a Vertical Pipe at constant velocities for each phase and various pressure conditions as shown in Fig. 4.

\subsubsection{Interfacial and Wall Friction Packages}

An interfacial friction factor accounts for interfacial force that can occur as a result of a momentum interchange between phases. Using flow regime information and interfacial area concentration, an interfacial friction factor is calculated. The interfacial area concentration for Taylor bubbles used in interfacial heat transfer calculation cannot be used for friction factor calculation because the area concentration for a drag model is the projected area of a Taylor bubble. For bubbly and slug flow conditions in a vertical channel, the drift flux model is used as the default model. The interfacial friction factor between a vapor and a droplet is also calculated. The interfacial friction coefficient has also been examined to ensure a smooth transition over all flow conditions as shown in Fig. 5.

For closing phasic momentum equations, a wall friction factor is also needed. The Churchill correlation [11] which is a fit to the Moody diagram is used to calculate
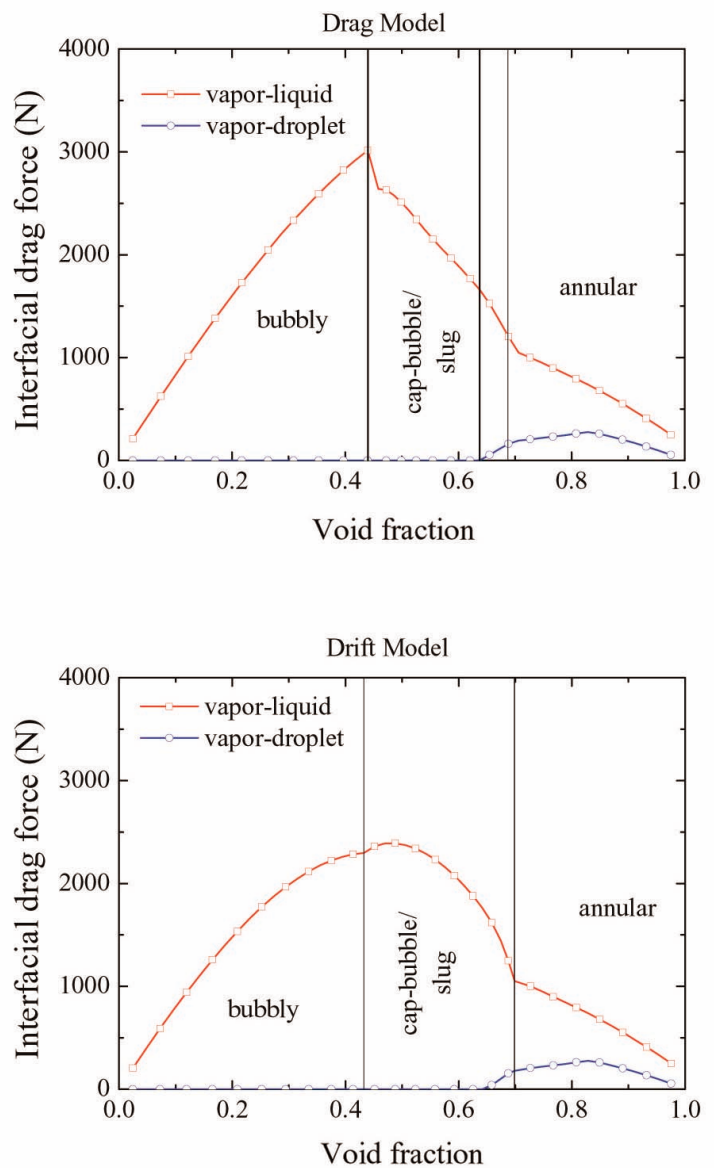

Fig. 5. Interfacial Drag Force as a Function of Void Fraction by Varying Flow Rate for Each Phase to Cross the Various Flow Regimes in a Vertical Pipe 
the wall friction factor for all flow regimes except for a stratified flow. The Churchill correlation was originally developed for a single phase flow. To account the twophase effect, a two-phase multiplier which is calculated from a modified Baroczy correlation [12] is used. To provide the pressure drop terms due to wall friction in momentum equations, the total two-phase friction pressure drop is partitioned into the wall friction pressure drops for continuous liquid, gas, and dispersed liquid fields by using a technique originally derived by Chisholm [13]. The theoretical basis for partition of the two-phase pressure drop is that the two-phase pressure drop for each field can be expressed in terms of pressure drops due to wall friction and interfacial friction by simplifing the momentum equations. Detailed derivation is decribed in the manual [28]. The SPACE code assumes that wall friction acts on each field independent of whether the field is in contact with a wall or not. For a horizontally stratified flow, the same Churchill correlation which is used in other flow conditions is used. The Reynolds number of each field for a horizontally stratified flow is calculated using the hydraulic diameter and the fluid properties of each field. The wall friction coefficient has also been examined to ascertain whether it covers the entire flow condition and whether a smooth transition can be ensured as shown in Fig. 6.

\subsubsection{Droplet Entrainment and De-Entrainment Package}

Droplet entrainment from a continuous liquid and deentrainment to a continuous liquid can occur in annularmist and horizontal stratified flows. The prediction of droplet behavior may affect an accurate calculation of the reflood phenomena. The Pan-Hanratty [14] model is chosen to calculate the entrainment rate for a horizontal annularmist flow because no other appropriate model has been found in the literature.

\subsubsection{Wall Heat Transfer Package}

The wall-to-fluid heat transfer modes consist of liquid-phase natural convection, liquid-phase forced convection, subcooled and saturated nucleate boiling, critical heat flux, transition boiling, film boiling, vaporphase convection, and condensation heat transfer. A total of 12 heat transfer modes have been determined. A heattransfer mode-transition map was developed to ensure a smooth transition between two adjacent heat transfer modes and to minimize the numerical instability. The heat-transfer mode-transition is based on pressure, noncondensible gas quality, void fraction, degree of subcooling, and wall temperature. The unique feature of a wall-to-fluid heat transfer package of the SPACE code is to adopt a lookup table method for a film boiling region rather than a conventional correlation method. The minimum film boiling temperature is used to define the boundary between transition and film-boiling heat-transfer modes. The heattransfer mode-transition map is shown in Fig. 7. The reflood heat-transfer package is incorporated into a heat structure model which takes account of the two-dimensional conduction effect. This package deals with the wall-tofluid heat transfer during the reflood phase as a separate model package. A heat flux partition into continuous liquid, dispersed liquid, and vapor fields is also taken into account to provide the source terms of the field equations.

\subsection{Special Process and Component Models}

To limit or modify the solution of the basic governing equations reflecting physical phenomena and to provide the capability to simulate the systems of nuclear power plants, special process and system component models are provided in the SPACE code.

\subsubsection{Special Process Models}

Special process models encompass choking of discharged or internal flow, limitation of countercurrent flow, off-take through the opening in a lateral direction in case of horizontal flow, water-level tracking in vertical cells, and the abrupt change of the flow area.

A two-phase choking model is developed based on Ransom and Trapp [15]. The choking criterion for a twophase three-field flow is derived by solving four simultaneous equations of total mass conservation, two phasic momentum conservations where droplet and continuous liquid together are treated as a liquid on the assumption of the same densities, and total energy conservation. Choking is determined to occur if the calculated criterion is equal to or larger than the sonic velocity of the homogeneous equilibrium model. Then the velocities calculated by the conservation equations are limited by the sonic velocity. In addition to this model, conservative models of Moody [16] and Henry-Fauske [17] are also implemented for their probable applications.

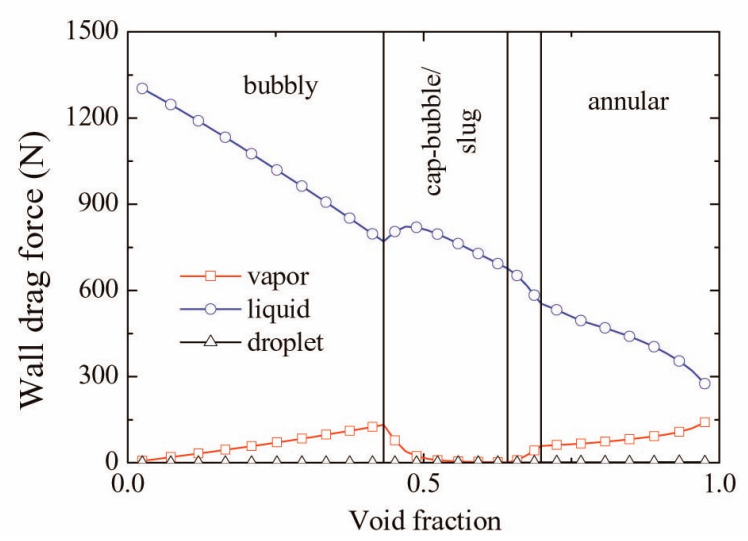

Fig. 6. Wall Frictional Force as a Function of Void Fraction by Varying Flow Rate for Each Phase to Cross the Various Flow Regimes in a Vertical Pipe 
Figure 8 shows a comparison of the pressures between the measurement and calculations by Trapp-Ransom models implemented in SPACE and in RELAP5 of Edward's pipe experiment [18]. The SPACE code compares very well with the measured data.

The limitation of countercurrent flow is modeled using Wallis [19], Kutateladze [20], and Bankoff [20] correlations. The activation of each of these models is controlled by user input. If the model is activated, the liquid downflow velocity calculated by the solver is replaced by the velocity of this limitation model.

An offtake model is used to improve the prediction of mass and energy flux through the opening lateral to the main flow direction in a horizontal flow. Four offtake models are implemented depending on water levels and opening locations. Top offtake and bottom offtake models are used to predict the water and gas entrainment, respectively. Two cases of side offtakes are modeled depending on the relative elevation of the water level and the opening. If the water level is higher than the opening elevation, gas entrainment is calculated and reflected in the calculations of the total opening flow rate. If the level is lower than the opening, the liquid entrainment is calculated and considered in the calculation of the total opening flow rate. Flow quality at the opening is determined as a function of the water level in the horizontal pipe.

A level-tracking model is used to avoid inaccuracy in the predictions of the flow map and of donor cell properties when a void profile shows discontinuity in a control volume. The level-tracking model is applied to a vertical cell or a series of vertical cells. In this model, each region of gas dominant and mixture dominant in a cell is separately treated. Detection of the two-phase level and modification of the conservation equations reflecting the existence of the level are important features of this tracking model.

\subsubsection{System Component Models}

System component models are given for the centrifugal pump, valves, pressurizer, safety injection tank, mixing of the emergency core cooling water (ECCW) and the

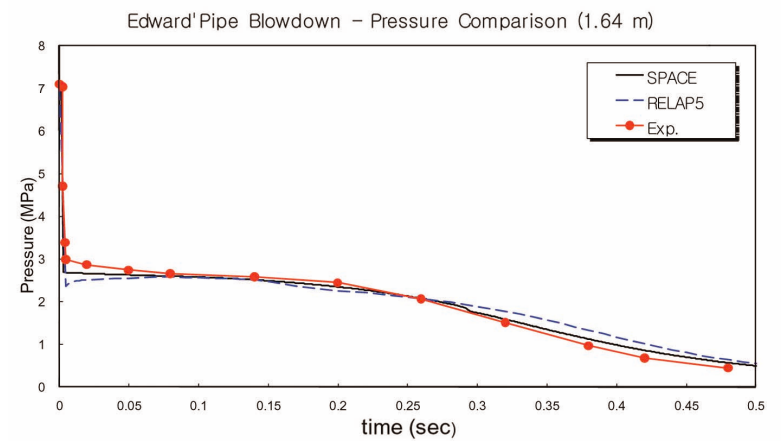

Fig. 8. Prediction of the Pressure for Edward's Pipe Problem

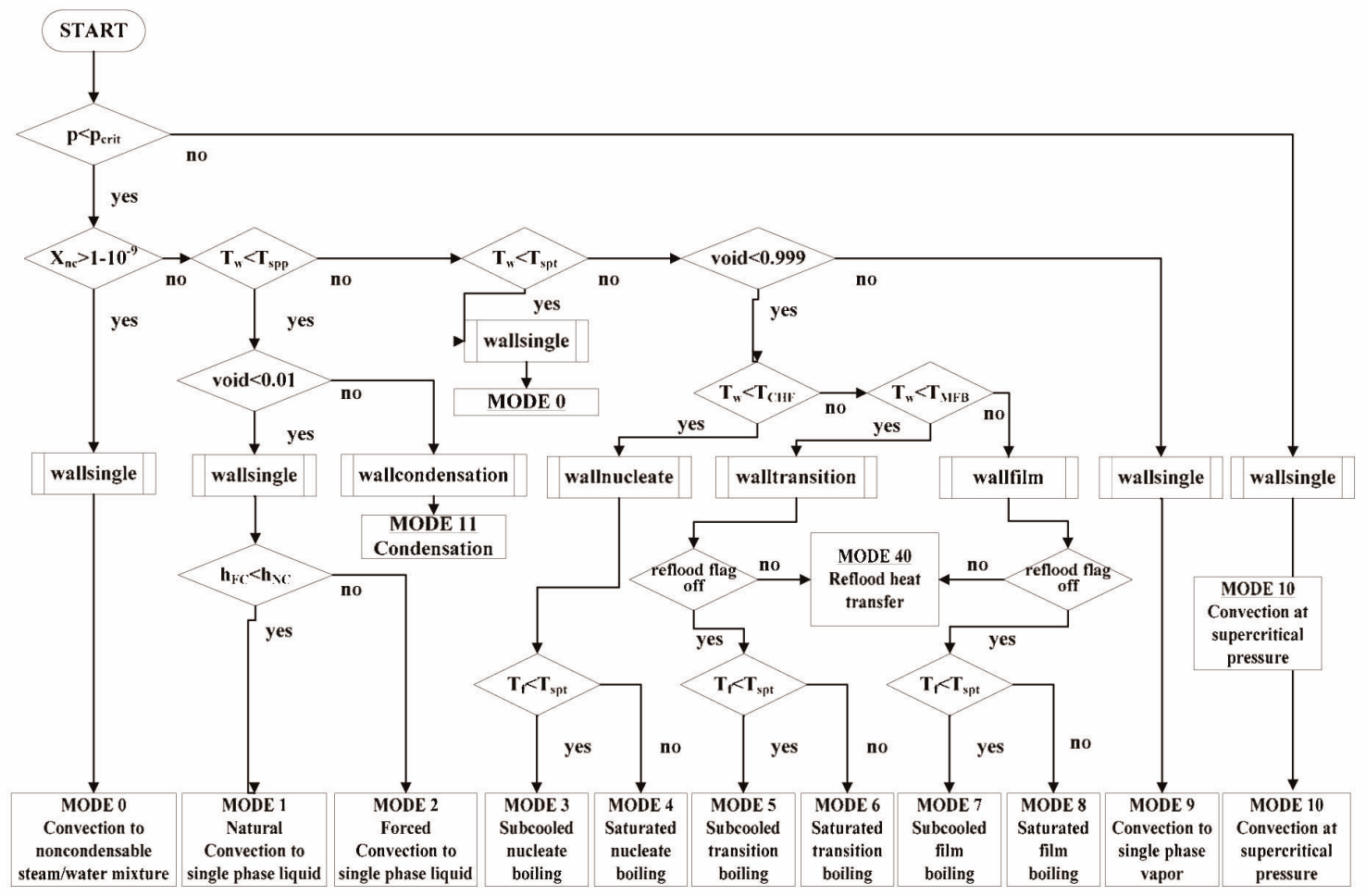

Fig. 7. Heat Transfer Mode-Transition Map 
steam, separator, and turbine.

The performance of the centrifugal pump is represented by homologous curves for a single-phase condition and differences for a two-phase condition. The independent variable of the curve is determined as a ratio of nondimensional values of flow and pump velocity, and then the dependent variable of the non-dimensional head or torque value is obtained from the homologous curves. The two-phase pump performance is calculated by a conventional method using degradation multiplication factors.

Several types of valves are grouped as trip valves, pressure valves, and time-rate valves in the SPACE code. Trip valves perform simple opening and closing of flow areas depending on user-specified conditions. They are set to be open afterwards when the condition is true; otherwise, they are assumed to be closed. This kind of valve can be utilized in conditions such as break opening and simple closing of feedwater line valves. Pressure valves are further grouped into three categories: first, check valves which open or close depending on the pressure difference between upstream and downstream, second, valves to simulate the flow area as a function of upstream pressure, and third, valves to model the pressure hysteresis effect. The second type can be utilized to model the opening of steam line safety valves as a function of upstream pressure. Hysteresis valves are modeled in a user-convenient manner. While conventional codes require complicated user inputs to model hysteresis, the SPACE model requires simple table inputs of flow area and upstream pressures. Characteristics of hysteresis shown in Fig. 9 are modeled as seven sets of pressure and flow area values. Time-rate valves are used to model the variation of flow area as a function of time or stem position. Four variations of valve operation are possible depending on open and close signals as shown below in Table 1. For all three types of valves, the leakage ratio, which is defined as the ratio of the remaining open area to the full valve area in case of closing, is taken into consideration to model the valve leakage due to aging or any malfunction.

The pressurizer component is a combination of pipe and special functions. The pressure control mechanism of heater and spray is explicitly modeled in addition to general heat structures. Heat addition and removal by heaters and sprays are simplified to be instantaneously distributed throughout the corresponding region of liquid or steam, respectively. Interfacial heat transfer between droplets and steam is calculated assuming that a saturation condition prevails in a pressurizer, and the only credible temperature difference is that of spray and surrounding steam. Decay and growth of spray droplets are not modeled, and the diameter of the spray droplets is assumed to be constant.

The safety injection tank is a specialized component which solves the conservation equations separately assuming full separation of cushion gas and liquid in a tank. Depending on the pressure difference between the tank and a downstream cell, the discharge mass flow rate is calculated, and the condition of the tank is calculated accordingly. Mixing of ECCW and steam in a cold leg is modeled as a special branch component which is activated only when the ECCW is available. Based on a previous study [21], two regimes of stratified flow and plug flow are modeled, and all other regimes conceptually possible but not observed are not considered. The criterion to distinguish the plug flow from the stratified flow is the thermodynamic ratio defined as condensation capacity of ECCW divided by steam condensation heat.

Two separator models are available in SPACE to separate steam from the two-phase mixture of the steam generator secondary side, one for simple application and the other for detailed mechanical modeling. Void fractions of steam outlet and water fall-back faces are determined

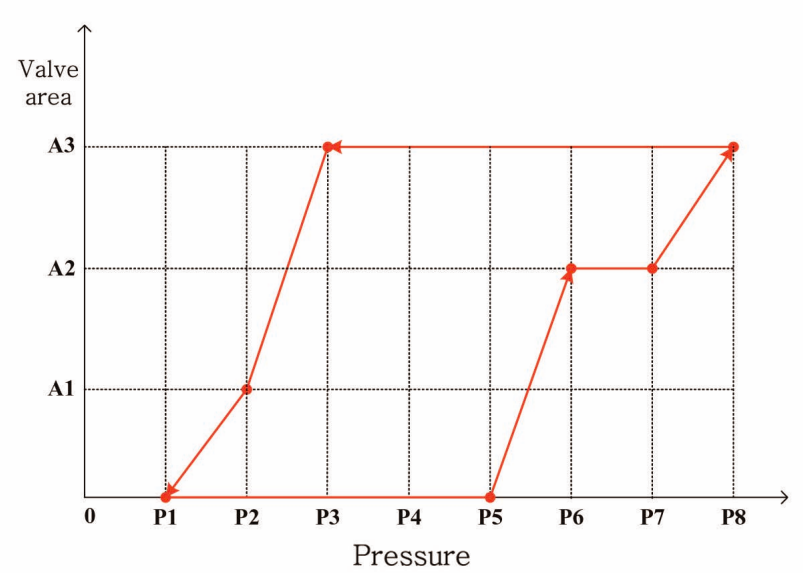

Fig. 9. Generalized Shape of Valve Hysteresis

Table 1. Modes of Time-Rate Valve Operation

\begin{tabular}{c|c|c|c}
\hline Case & OPEN & CLOSE & Operation \\
\hline 1 & TRUE & TRUE & Error, end \\
\hline 2 & TRUE & FALSE & Valve opens as a function of user table or rate values \\
\hline 3 & FALSE & TRUE & Valve closes as a function of user table or rate values \\
\hline 4 & FALSE & FALSE & Valve stays, previous flow area is maintained. \\
\hline
\end{tabular}


as a simple function of the void fraction in the separator cell in a simple model, while the void fractions are calculated considering the centrifugal forces of droplets passing swirl vanes in vortex chambers and the geometric configuration of the chambers in a detailed mechanical model. Two turbine models are implemented in the SPACE code, one for simple application and the other for a detailed model. In the simple model, turbine stages are modeled as a single stage, and efficiency and turbine power are calculated using the pressure difference between the inlet and outlet. In the detailed model, the radius, reaction ratio, and blade velocity of each stage are determined using user-supplied geometry inputs and applying general pressure ratios.

\subsection{Heat Structure Model}

One- and two-dimensional heat conduction models are implemented in SPACE. The one-dimensional model can treat geometries of rectangular slab, cylinder, and sphere. In this one-dimensional model, the temperature variation along the thickness or radial direction is calculated assuming symmetries and/or ignoring variations in other directions. The two-dimensional model is implemented to calculate two-dimensional temperature distribution of a fuel rod in axial and radial directions, especially in case of reflooding. The cylindrical geometry is exclusively considered, and symmetry in the azimuthal direction is assumed. If reflood calculation is activated, and the temperature difference between neighboring mesh points in the axial direction exceeds a user-specified value, heat conduction meshes in the axial direction are reconstructed into more detailed meshes to capture relatively large temperature differences in very short axial distances.

Nuclear fission heat of a nuclear fuel rod is calculated by a point kinetics equation and is treated as a heat source in the heat conduction equation. Reactivity feedbacks of moderator density, moderator temperature, fuel temperature, boron concentration, reactor scram, and power defect are considered, and decay heat of ANS-73, -79 and -94 models are implemented. Conductivity of the gap between a fuel pellet and cladding is calculated considering the conduction of the gap gas mixture, solid contact conduction, and the radiation across the gap. Five kinds of gap gases, namely, helium, argon, krypton, xenon, and nitrogen are considered.

Mechanical and thermal deformation of a fuel rod has detrimental effect on the gap conductance and the resulting cladding temperatures. Fuel deformation is divided into that of the pellet and that of the cladding. Pellet deformation is assumed to be caused by thermal expansion, and the effects of fuel relocation and swelling or densification of the pellet triggered by fission gases are not modeled. They are assumed to be modeled by pellet property inputs such as conductivity and volumetric heat capacity. Cladding deformation is divided into thermal expansion, elastic deformation, plastic deformation, and rupture, and these four mechanisms are all considered. Plastic deformation is modeled as a function of rupture strain and temperature, which are functions of hoop stress on the cladding. Cladding rupture is assumed to occur when the average temperature of the cladding is higher than the rupture temperature, and the resultant rupture strain is calculated by the NUREG-0630 [22] model. Another important model is of metal-water reaction. The reaction thickness is calculated using the Cathcart-Pawell model [23]. The oxidation is calculated only onto the outside surface of the cladding until the cladding is ruptured, afterwards, oxidation of both sides is considered. Exothermic reaction heat is treated as an additional heat source for the heat conduction equation. Material properties frequently used in nuclear power plants, such as $\mathrm{UO}_{2}$, Zircaloy, Inconel, and so on, are implemented in the code for user convenience. Radial temperature distribution of a fuel rod calculated by the SPACE code is shown in Fig. 10 . Abscissa of the figure is the mesh point number. The typical temperature gradients across fuel pellet, fuel gap, and cladding are found to be well predicted.

\section{CODE ASSESSMENT}

Code assessment has been performed in terms of several conceptual problems and separate effect test problems generally used in conventional safety analysis code assessment [6-10]. The results were compared with analytic or experimental data.

\subsection{Nine-Volume Water Over Steam}

This test problem is designed to check the gravitational head effect and the development of countercurrent flow from an initial sharp liquid-vapor interface. The vertical pipe which has a length of $4.165 \mathrm{~m}$ and an area of $1.0 \mathrm{~m}^{2}$ was modeled using nine volumes and eight junctions. The upper one-third of the pipe was initially filled with saturated water, and the bottom two-thirds of the pipe

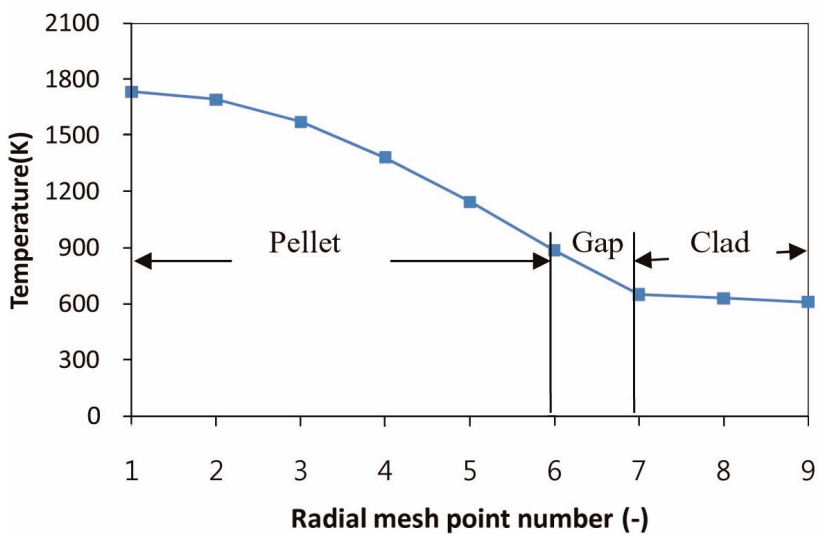

Fig. 10. Radial Temperature Distribution of a Fuel Rod Calculated by the SPACE Code 
was filled with saturated steam at $413 \mathrm{kPa}$. Figure 11 shows the distribution of the void fraction at various times during the test. The void fraction in the intermediate volumes drops to around 0.80 during the liquid fall down, as shown in the distribution of the void fraction at 6 seconds. This indicates that the liquid forms a thin stream as it flows to the bottom volumes. The overall behavior of void fraction at various elevations indicates the interphase friction models of SPACE allow the liquid to fall to the bottom of the pipe in a smooth manner. The bottom node is full of liquid at a time earlier than 2 seconds. This demonstrates that the calculated gravitational effect and kinematic models for phase continuity provide a qualitatively correct result.

\subsection{Nitrogen-Water Manometer Problem}

A 20-volume nitrogen-water manometer was set up to check the non-condensable gas state calculation and the code momentum formulation for periodic flow. Each volume had an area of $0.01 \mathrm{~m}^{2}$ and a length of $1.0 \mathrm{~m}$. The first 10 volumes were oriented vertically downward and the last 10 volumes were oriented vertically upward. Pressure boundary conditions of $2 \mathrm{MPa}$ are given at both the pipe inlet and outlet. To initiate the oscillation by

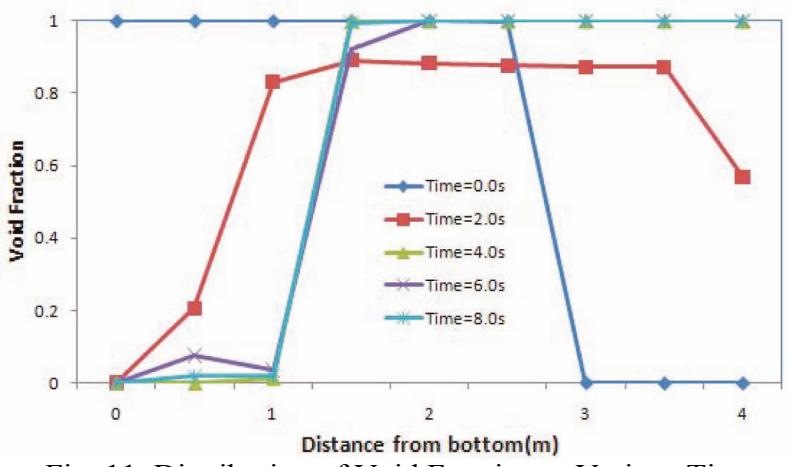

Fig. 11. Distribution of Void Fraction at Various Times

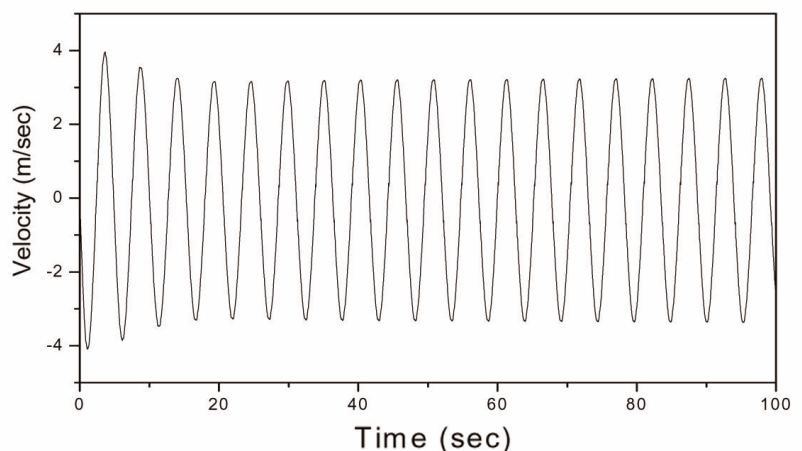

Fig. 12. Liquid Velocity at the Bottom of the Nitrogen-Water Manometer head difference, the bottom seven volumes on the left and the bottom three volumes on the right side were filled initially with water at $2 \mathrm{MPa}$ and $350 \mathrm{~K}$. The remaining volumes were initialized with dry nitrogen at the same pressure and temperature. When the test started, the liquid oscillated back and forth between the two vertical columns. The calculated period of oscillation by the SPACE code is about 4.8 seconds, which seems reasonable compared to the theoretical value of 4.6 seconds, calculated for this conceptual problem with the assumption of neither wall nor interfacial frictions.

\subsection{Branch Reentrant Tee Problem}

The branch reentrant tee problem is comprised of two identical horizontal pipes, a tee branch, and a horizontally oriented single-cell component as shown in Fig. 13. All the pipe area is $1.0 \mathrm{~m}^{2}$, and the length of each cell is 1.0 m. Two horizontal pipes (components 200 and 300) are connected to the outlet side of a horizontally oriented branch component (100) which represents the tee. The same pressure boundary conditions are given to the upstream side of each of the pipes. A horizontally oriented single cell (400) is also connected to the outlet side of the branch component. A pressure boundary condition is then given to the downstream side of the single cell. The fluid conditions set in the supply sources (201 and 301) and all components are single-phase, saturated water, at $2.64 \mathrm{MPa}$ and $500 \mathrm{~K}$. The fluid conditions set in the outlet pressure boundary (401) are single-phase saturated water at 2.5 $\mathrm{MPa}$ and $497.1 \mathrm{~K}$. Theoretically, the flows through the pipes should be symmetrical and the outflow to the outlet pressure boundary should be the sum of the two pipe flows. The results are shown in Table 2. The symmetrical flow in the two pipes upstream from the modeled tee component (branch) was calculated by the SPACE code. The difference in the total mass flow is insignificant.

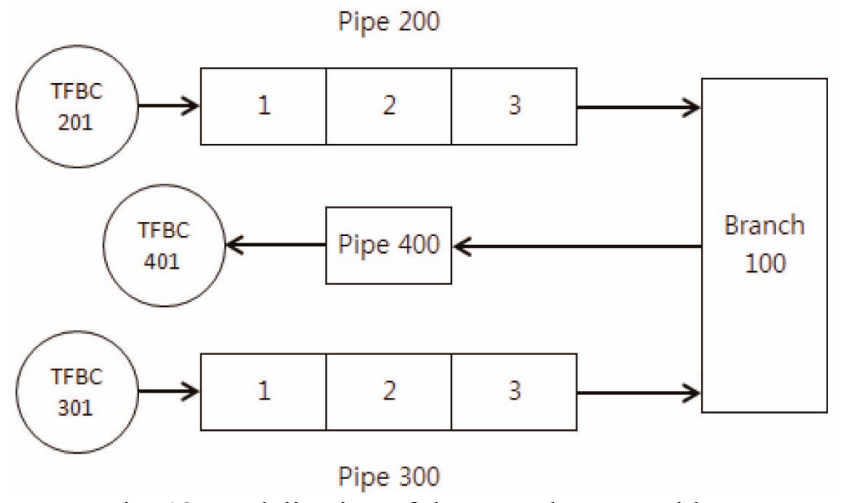

Fig. 13. Nodalization of the Branch Tee Problem 


\subsection{Cross-Flow Tee Problem}

The model nodalization for this problem is similar to the model of the branch tee problem, except that the outflow is modeled using a vertical single cell (component 400). The same pressure boundary conditions are given to the upstream side of each of the pipes. A pressure boundary condition is then given to the downstream side of the single cell. The fluid conditions were the same as the branch-tee model presented previously. Theoretically, the flows through the pipes should be symmetrical, and the outflow to the outlet pressure boundary should be the sum of the two pipe flows. As shown in Table 3, the calculated discharge mass flow rate agrees well with the combined mass flow rates of the two upstream pipes. It is demonstrated with this problem that crossflow junctions can be used to model the behavior of a tee without encountering unphysical behavior.

\subsection{Cross Tank Problem}

The cross tank problem is a conceptual problem that was set up to test for flow anomalies that may appear as recirculating two-phase or single-phase flows using the cross-flow junction feature in the code to model multidimensional effects. The cross tank model consists of two vertically oriented pipe components equally divided

Table 2. Results for the Branch Tee Problem

\begin{tabular}{c|c}
\hline Variables & Values at 20 seconds \\
\hline Mass Flow Rate $(201)(\mathrm{kg} / \mathrm{s})$ & 2666.70 \\
\hline Mass Flow Rate $(301)(\mathrm{kg} / \mathrm{s})$ & 2666.70 \\
\hline Mass Flow Rate $(401)(\mathrm{kg} / \mathrm{s})$ & 5333.50 \\
\hline Sum of $201 \& 301(\mathrm{~kg} / \mathrm{s})$ & 5333.40 \\
\hline Difference $(\%)$ & 0.0019 \\
\hline Mass Error $(\mathrm{kg})$ & 0.0746 \\
\hline
\end{tabular}

Table 3. Results for the Crossflow Tee Problem

\begin{tabular}{c|c}
\hline Variables & Values at 20 seconds \\
\hline Mass Flow Rate $(201)(\mathrm{kg} / \mathrm{s})$ & 3591.00 \\
\hline Mass Flow Rate $(301)(\mathrm{kg} / \mathrm{s})$ & 3591.00 \\
\hline Mass Flow Rate $(401)(\mathrm{kg} / \mathrm{s})$ & 7180.94 \\
\hline Sum of 201 \& 301 (kg/s) & 7182.00 \\
\hline Difference $(\%)$ & 0.0148 \\
\hline Mass Error $(\mathrm{kg})$ & 0.1179 \\
\hline
\end{tabular}

into 19 cells. Cross-flow junctions connected the two pipe components at each cell. The bottom 15 cells in each pipe component were initialized with water at $0.1014 \mathrm{MPa}$ and $305 \mathrm{~K}$. Cell 16 in each pipe component contained a mixture of air and water in equilibrium condition with a static quality of 0.5 and a pressure and temperature the same as liquid. The remaining three top cells of each pipe component were in equilibrium conditions. The problem was run using null flow conditions (zero velocity). The expected result was that the system would remain near the null condition throughout the calculation.

The calculated crossflow junction mass flow rates just below and above the liquid level in each of the two pipe components are shown in Fig. 14. It can be seen that the cross-flow junction mass flow rates remain near zero, demonstrating that there were no non-physical recirculation flows caused by the SPACE single- or two-phase flow models.

\subsection{Horizontally Stratified Countercurrent Flow}

The horizontally stratified countercurrent flow problem consists of a horizontal pipe closed at both ends with a linearly graduated liquid level. Because of the gravitational head difference, the liquid tends to flow from the higherlevel side to the lower-level side due to the hydrostatic pressure difference caused by stratification and the vapor flows in the opposite direction. The problem was Selected to verify that the speed of propagation for a void wave is comparable with the theoretical value. The pipe was modeled using 20 volumes (total pipe length $10 \mathrm{~m}$, flow area $0.19635 \mathrm{~m}^{2}$ ). The pipe was initially filled with a

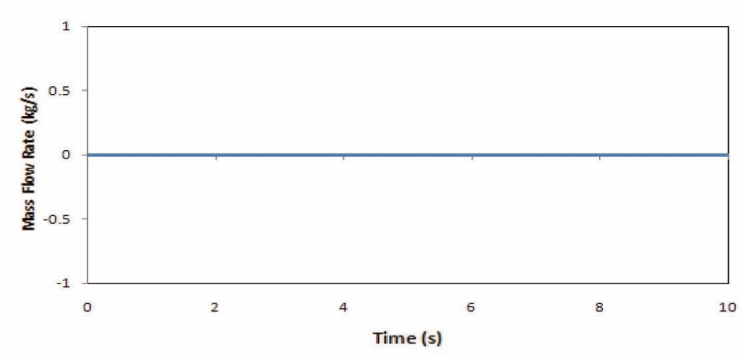

(a) at above liquid level (gas region)

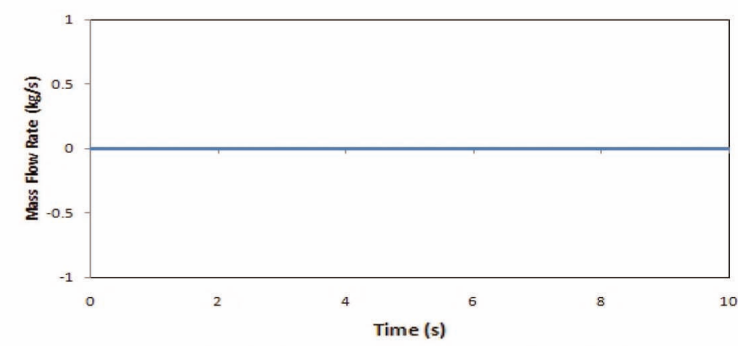

(b) at below liquid level (liquid region)

Fig. 14. Mass Flow Rate of the Crossflow Junction 
saturated two-phase mixture at a pressure of $10 \mathrm{MPa}$; and the void fraction varied from 0.53 to 0.47 . The propagation speed from the SPACE calculated result was $1.4 \mathrm{~m} / \mathrm{s}$, while the theoretical value for a stratified wave in frictionless flow is $1.381 \mathrm{~m} / \mathrm{s}$. These results show that the horizontal stratified flow model in SPACE is functioning correctly.

\subsection{Multi-Dimensional Problems}

A test problem for the single-phase liquid flow in a two-dimensional horizontal cavity is designed to check the multi-dimensional flow models including multidimensional formulation of the momentum equations. The test domain consists of 102 uniform regular hexahedral cells with a side length of $1.0 \mathrm{~m}$. Initially, the rectangular cavity was filled with subcooled liquid at $1 \mathrm{MPa}$ and 300 $\mathrm{K}$. The inlet boundary condition was given at the lower left corner of the rectangular cavity, and the outlet boundary condition was given at the upper right corner: the inlet flow velocity was $0.2 \mathrm{~m} / \mathrm{s}$, and the outlet pressure was $1 \mathrm{MPa}$.

Figures 15 and 16 show the velocity vectors and the pressure distribution, respectively. The cavity flow was mainly formed along the bottom and the right side walls.
Actually, the word "bottom" here does not mean the physically lowest wall; rather, it means the wall that looks as if it is located lowest in the following illustration of cavity. The remaining part of the cavity shows a swirl flow pattern. The pressure distribution in the cavity shows the maximum pressure (red color) at the lower right corner, and almost uniform pressure distribution in the swirling region. Commercial computational fluid dynamics (CFD) codes such as STAR-CD [24] have much more accurate multidimensional flow models than the SPACE code which does not have momentum diffusion terms and turbulence models. However, when the same level of coarse meshes are used, the cavity flow patterns and pressure contours calculated by SPACE look similar to those of the well-known commercial CFD code, STAR-CD, indicating that the SPACE multi-dimensional flow models works properly.

A two-phase cavity flow test was also performed. The test geometry is the same horizontal cavity as in the multi-dimensional single-phase flow test. However, the test domain consisting of 10x10 hexahedral cells was initially filled with saturated vapor at $0.1 \mathrm{MPa}$. When the test started, saturated liquid at the same pressure was injected at the lower left corner with the velocity of 0.2

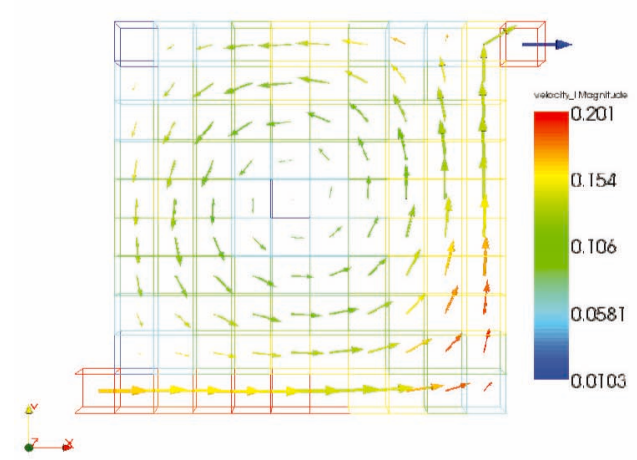

(a) SPACE

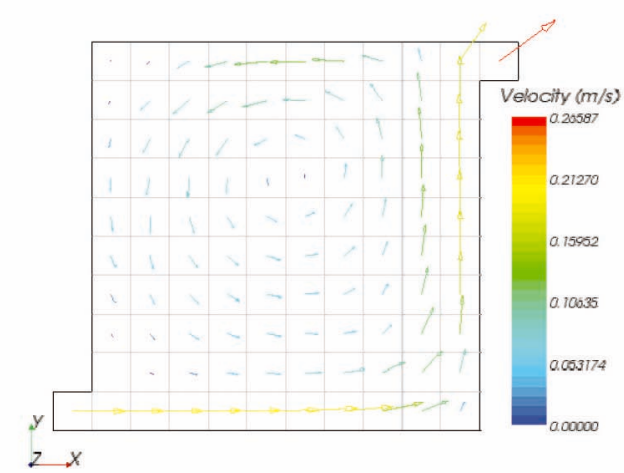

(b) STAR-CD

Fig. 15. Velocity Vectors for Single-Phase Cavity Flow
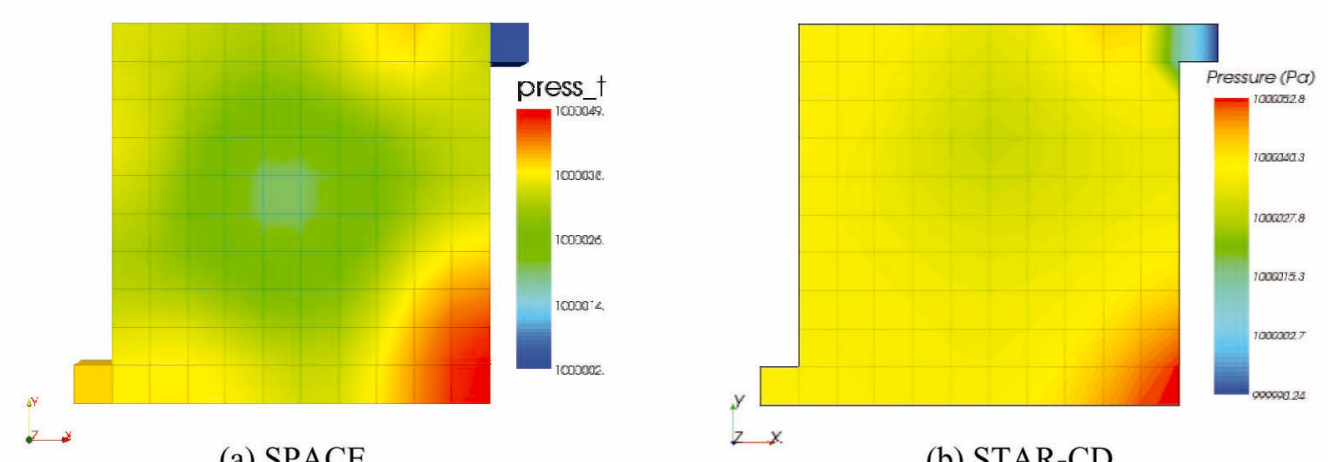

Fig. 16. Pressure Contours for Single-Phase Cavity Flow 
$\mathrm{m} / \mathrm{s}$. Figure 17 shows the void fraction distribution at 200 seconds. Comparison with the STAR-CD result shows that the SPACE multi-dimensional two-phase model reasonably simulates the multi-dimensional two-phase flow phenomena.

Then, a test problem of multi-dimensional flow in a vertical annulus was designed to check the code capability to analyze two-phase flow in the cylindrical geometry. The annulus shown in Fig. 18 was initialized with pure vapor slightly superheated at 1.0 MPa, and then the liquid slightly subcooled at the same pressure started to fill from the two injection nozzle. Each of the liquid injection nozzles is located at the opposite side of the medium level outer wall. A broken nozzle and a vapor injection nozzle were also aligned at the same level 90 degrees apart from the liquid injection nozzles in the azimuthal direction. The test result shows that liquid film penetrates to the bottom of the annulus, in spite of the vapor flow crossing the liquid flow. Once the liquid fills up to the level of the broken nozzle, the remaining liquid spills out except the near region of the vapor injection nozzle, where liquid droplet or liquid film is driven to the top of the annulus due to the strong vapor flow. The overall behavior of the liquid injection flow, lateral vapor flow, and their interactions seem to be quite reasonable qualitatively.

\subsection{GE Level Swell}

Level swell is a phenomena occurring in depressurization events. To correctly predict level swell phenomena, accurate flashing and interface drag models are required. Two different experiment facilities consisted of a 0.3048 $\mathrm{m}(1 \mathrm{ft})$ diameter tank, designated the small vessel, and a $1.2192 \mathrm{~m}(4 \mathrm{ft})$ diameter tank, designated the large vessel [25]. Before experiments started, the vessels were heated to obtain experimental pressure and temperature. Then, experiments were started by releasing the pressure of the vessels. The boundary conditions for pressure histories were simulated by adjusting the discharge coefficient of the break valve. Pressure calculation using $\mathrm{Cd}=0.85$ gives the best boundary conditions for pressure transient. The experimental void fractions used for comparisons with MARS and SPACE predictions were calculated from the delta P measurements. The test 1004 for the $0.3048 \mathrm{~m}$ diameter vessel and test 5801 for the $1.2192 \mathrm{~m}$ diameter vessel were simulated. Comparisons from 1004-3 and

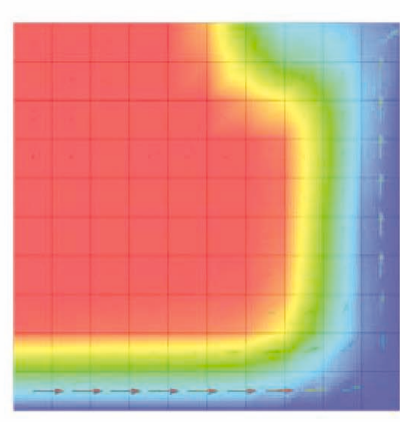

(a) SPACE

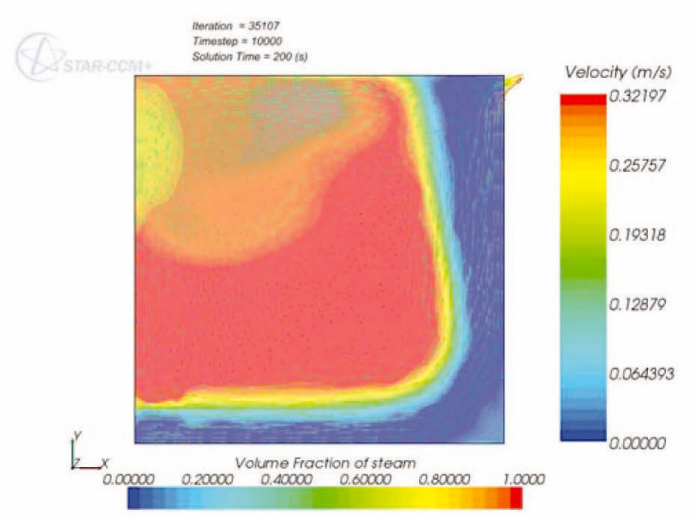

(b) STAR-CD

Fig. 17. Void Fraction Contours at 200 Seconds For the Two-Phase Cavity Flow Test
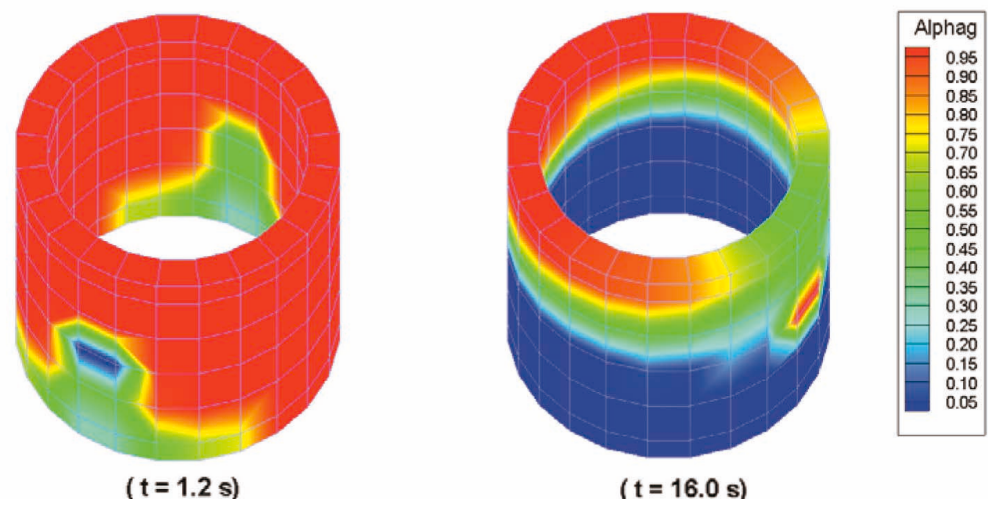

Fig. 18. Distribution of Void Fraction During the Two-Phase Annulus Flow Test 

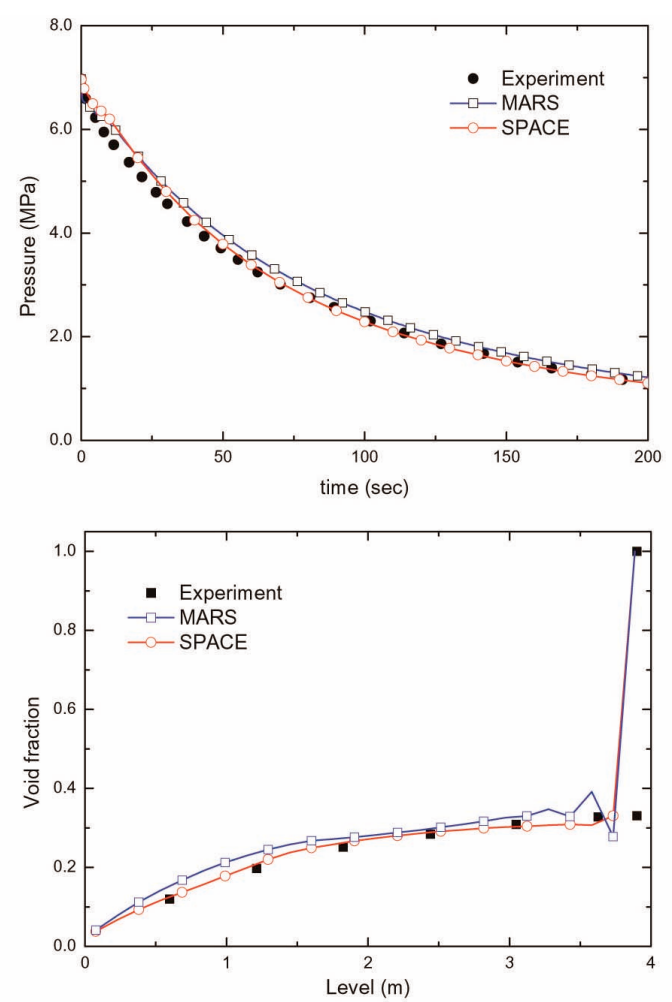

Fig. 19. Comparisons of the Calculated Results with Experiments for GE 1004-3

5801-15 are shown in Figs. 19 and 20. The calculated void profile from the small and large vessel comparison is in good agreement with the measured data.

\subsection{Bennett's Heated Tube Experiments}

The Bennett heated tube experiments [26] were conducted using a vertical $1.26 \mathrm{~cm}$ diameter tube that was electrically heated. Water at a pressure of $6.9 \mathrm{MPa}$ flowed upward in the tube. The test section of the Bennett experiment was modeled with a pipe with 32 cells and a heat structure with 32 axial meshes. The calculated wall temperatures from SPACE, MARS, and TRACE are compared with the Bennett test data for the low, intermediate, and high mass flux tests in Figures 21 through 23. In general, the calculated wall temperatures and CHF locations are reasonable compared to the data for all three test cases. For the low mass flux case, the results matched the CHF location and the post-CHF wall temperature quite well. The other two cases show the CHF location occurring at a higher elevation (Test 5294) or at a lower elevation (Test 5394). The wall temperature for the post-CHF condition was reasonably predicted for the low and intermediate mass flux cases. However, for the high mass flux case, the wall temperature was overpredicted. This may be due to less droplet entrainment at high flow condition and ignoring the heat transfer
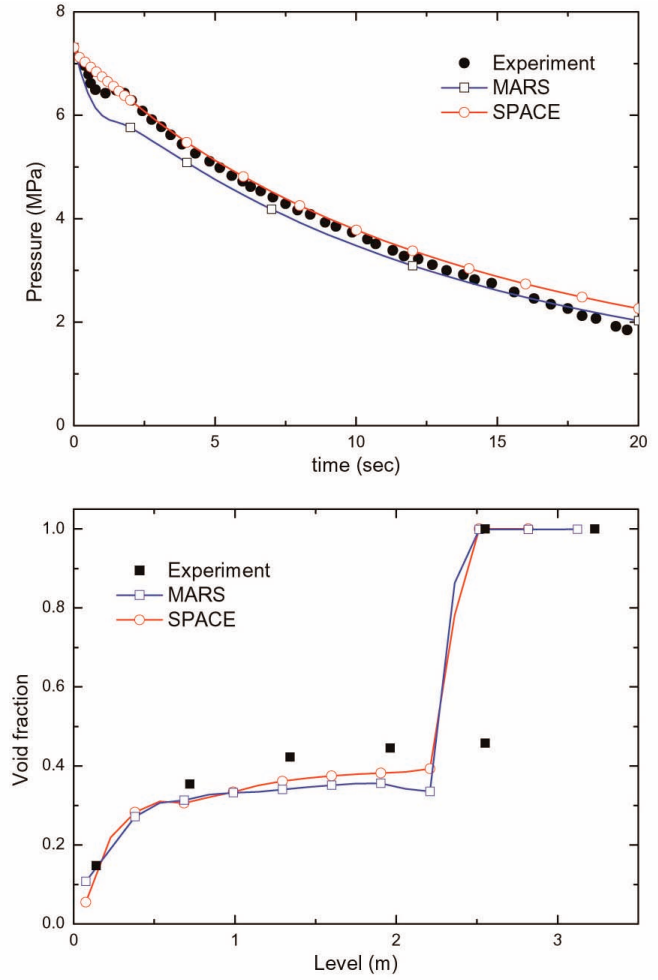

Fig. 20. Comparisons of the Calculated Results with Experiments for GE 5801-15

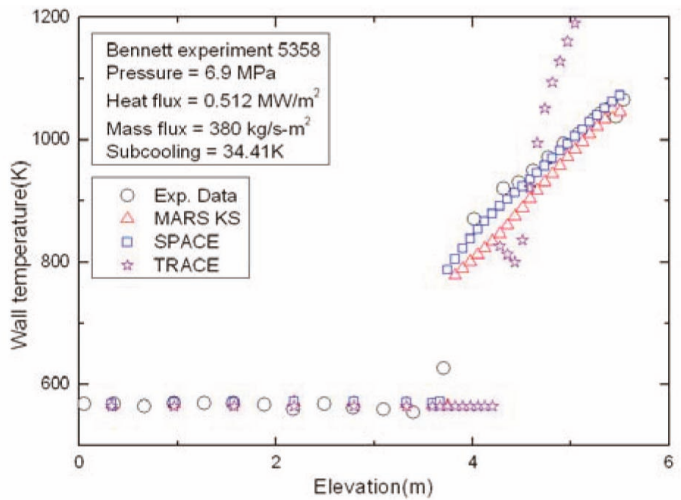

Fig. 21. Wall Temperature Profiles for Low Flow Case: Test 5358

enhancement in the developing film boiling region because the look-up table for film-boiling heat transfer is developed based on fully developed film boiling [27].

\section{CONCLUSIONS}

The Korean nuclear industry has been developing SPACE code for safety analysis of operating PWRs and new advanced reactor designs. The SPACE code adopts advanced physical modeling of two-phase flows, mainly two-fluid, three-field models, and has the capability to 


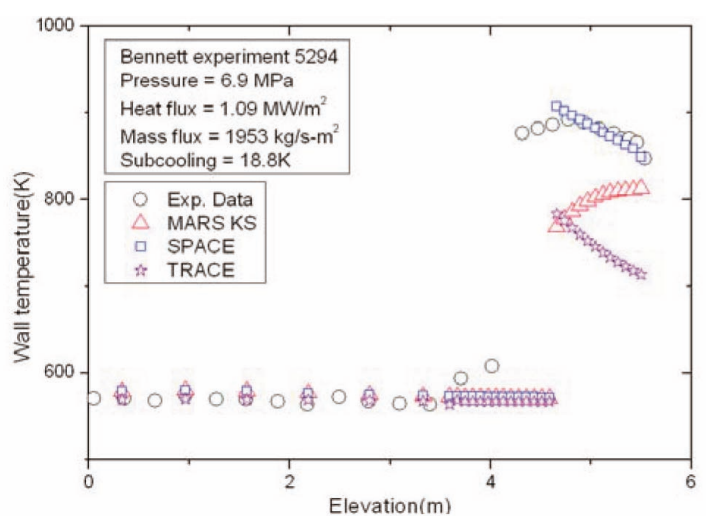

Fig. 22. Wall Temperature Profiles for Intermediate Flow Case: Test 5294

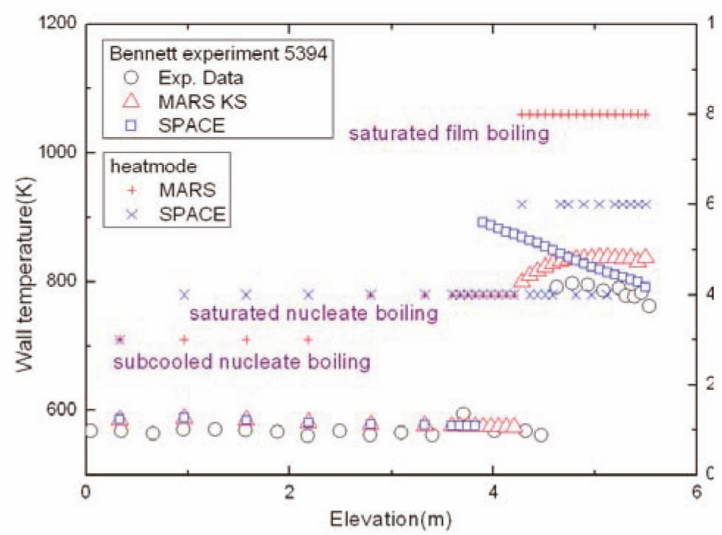

Fig. 23. Wall Temperature Profiles for High Flow Case: Test 5394

simulate 3D effects by the use of structured and/or nonstructured meshes. The SPACE code has up-to-date constitutive models incorporating a dispersed liquid field in addition to vapor and continuous liquid fields and contains special process and system component models to provide the capability to simulate the systems of nuclear power plants. These characteristics of SPACE code can help improve the accuracy of the prediction of nuclear reactor system behavior under transient conditions. Code assessments using conceptual problems and separate effect test problems were performed and showed good results. As a result of the SPACE code development project, a demo version was released in March 2010. Further code validation activities according to the SPACE code V\&V matrix [29] are scheduled for the end of 2012, and an evaluation version is planned for the end of 2011.

\section{ACKNOWLEDGEMENTS}

The SPACE code has been jointly developed by Korea Electric Power Corporation Research Institute (KEPRI), KEPCO Engineering \& Construction Company(KEPCO E\&C), Korea Atomic Energy Research (KAERI), Korea Nuclear
Fuel (KNF) and Korea Hydro \& Nuclear Power Co., LTD (KHNP), and this work has been supported by the Ministry of Knowledge Economy (MKE) of the Republic of Korea.

\section{NOMENCLATURE}

$$
\begin{aligned}
& A \\
& \mathbf{B} \\
& C \\
& C \\
& \text { d } \\
& e
\end{aligned}
$$

$F_{g l} \quad$ : interfacial drag coefficient between liquid and vapor phase

$F_{g d} \quad$ interfacial drag coefficient between drop and vapor phase

$F_{w g} \quad:$ wall drag coefficient for vapor phase

$F_{w l} \quad:$ wall drag coefficient for liquid phase

$F_{w d} \quad$ : wall drag coefficient for drop phase

$l_{P}^{E} \quad:$ conversion factor of face direction to the outward direction from the present cell

$h \quad$ : enthalpy

n : face normal vector

$Q_{i v-1} \quad$ : heat transfer rate from the liquid-vapor interface to vapor phase

$Q_{i l} \quad$ : heat transfer rate from the liquid-vapor interface to liquid phase

$Q_{i v-d} \quad$ : heat transfer rate from the drop-vapor interface to vapor phase

$Q_{i d} \quad:$ heat transfer rate from the drop-vapor interface to liquid phase

$Q_{l-n} \quad$ : heat transfer rate from the liquid to noncondensable gas

$Q_{d-n} \quad$ : heat transfer rate from the drop to noncondensable gas

$S_{E} \quad$ : entrainment rate

$S_{D} \quad$ : de-entrainment rate

U : velocity vector

XU : cross-flow velocity

$U_{n} \quad$ : face normal velocity

$V \quad$ : volume

$\alpha \quad$ : void fraction

$\varepsilon \quad$ : porosity

$\psi \quad$ : transport property

$\rho \quad:$ density

$\Gamma_{l} \quad:$ net vaporization rate at the liquid-vapor interface

$\Gamma_{l, E} \quad$ : vaporization rate at the liquid-vapor interface

$\Gamma_{l, C} \quad$ : condensation rate at the liquid-vapor interface

$\Gamma_{d} \quad$ : vaporization rate at the droplet-vapor interface

$\Gamma_{d, E} \quad$ : vaporization rate at the drop-vapor interface 
$\Gamma_{d, C} \quad$ : condensation rate at the drop-vapor interface

$$
\begin{array}{ll}
\text { Subscript; } \\
d & : \text { dispersed liquid (drop) phase } \\
E & : \text { face indicator } \\
g & : \text { gas phase } \\
k & : \text { face type, } 1: \text { x-directional face, } 2: \text { y-face, } 3: \text { z-face } \\
l & : \text { continuous liquid phase } \\
m & : \text { mixture } \\
n & : \text { non-condensable phase } \\
N & : \text { neighbor cell adjacent to the present cell } \\
P & : \text { present cell } \\
v & : \text { vapor phase } \\
\phi & : \text { phase indicator }
\end{array}
$$

\section{Superscript;}

Bhcell: back half cell

$$
\begin{array}{ll}
d & : \text { donor cell indicator } \\
E & : \text { face indicator } \\
\text { Fhcell } & : \text { front half cell } \\
n & : \text { advanced time }
\end{array}
$$

neighbor: the adjacent cell to which a face is directed

owner : the adjacent cell from which a face originates

$s \quad$ : saturated state

\section{REFERENCES}

[ 1 ] S. Y. Lee et al., "Formulation of time and volume averaged two-fluid model considering structural materials in a control volume," Nuclear Engineering and Design, Vol. 239, Issue 1, pp 127-139, January 2009.

[2] S.V. Patankar, Numerical heat transfer and fluid flow, Hemisphere Publishing Corporation. 1981.

[3 ] C.Hirsch, Numerical computation of internal and external flows, John Wiley \& Sons, 1991.

[4] C. E. Park et al., "A Two-Fluid, Three-Field Hydraulic Solver for the Safety Analysis Code, SPACE," ANS winter meeting, Washington D. C., November 2009.

[ 5 ] D.R. Liles and W.H. Reed, "A semi-implicit method for two-phase fluid dynamics", J. Com. Phys., vol. 26, pp. 390-407, 1978.

[6] RELAP5/MOD3.3 Code Manual, Volume I: Code Structure, System Models and Solution Methods, NUREG/CR5535/Rev 1, December, 2001.

[ 7 ] TRAC-M/FORTRAN90 (VERSION 3.0) Theory Manual, LA-UR-00-910, July, 2000.

[ 8 ] Analysis of FLECHT SEASET 163-Rod Blocked Bundle Data Using COBRA-TF, NUREG/CR-4166, January. 1986.

[9] M. Robert, M. Farvacque, M. Parent and B. Faydide, CATHARE2 V2.5: a fully validated CATHARE2 version for various applications, Proceedings of the 10th International Topical Meeting on Nuclear Reactor Thermal Hydraulics (NURETH-10) Seoul, South Korea, October 5-9, 2003.
[10] MARS code manual, volume 1: code structure, system models, and solution methods, KAERI/TR-2812/2004, July 2006.

[11] Churchill, S. W., "Friction-Factor Equation Spans All FluidFlow Regimes," Chemical Engineering, pp.91 92., 1977.

[12] Claxton, K. T., Collier, J. G. and Ward, J. A., "H.T.F.S. Correlation for Two-Phase Pressure Drop and Void Fraction in Tubes," HTFS Proprietary Report HTFS-DR-28, AERE-R7162, 1972.

[13] Chisholm, D., "A Theoretical Basis for the LockhartMartinelli Correlation for Two-Phase Flow," Int. J. Heat and Mass Transfer, vol.10, pp.1767-1778, 1967.

[14] Pan, L. and Hanratty, T. J., "Correlation of entrainment for annular flow in horizontal pipes," Int. J. Multiphase Flow, vol.28, pp.385 408., 2002.

[15] J. A. Trapp and V.H. Ransom, A Choked-Flow Calculation Criterion for Nonhomogeneous, Nonequilibrium, TwoPhase Flows, Int. J. Multiphase Flow, Vol. 8, No. 6, pp. 669-681, 1982.

[16] F. J. Moody, Maximum Flow Rate of a Single Component, Two-Phase Mixture, Trans. of the ASME, J. of Heat Transfer, pp. 134-142, February 1965.

[17] R. E. Henry and H. K. Fauske, The Two-Phase Critical Flow of One-Component Mixtures in Nozzles, Orifices, and Short Tubes, Trans. of the ASME, J. of Heat Transfer, pp. 179-187, May 1971.

[18] A. R. Edwards and T. P. O'Brien, Studies of Phenomena Connected with the Depressurization of Water Reactors, Journal of the British Nuclear Energy Society, 9, pp. 125$135,1970$.

[19] G. B. Wallis, Flooding Velocities for Air and Water in Vertical Tubes, UKAEA, AEEW-R-123, 1961.

[20] S. G. Bankoff and S. C. Lee, A Brief Review of Countercurrent Flooding Models Applicable to PWR Geometries, Nuclear Safety, Vol. 26, No. 2, pp. 139-152, March-April 1985.

[21] U.S. Nuclear Regulatory Commission, Reactor Safety Issues Resolved by the 2D/3D Program, NUREG/IA-0127, GRS101. MPR-1346, July 1993.

[22] D. A. Powers and R. O. Meyer, Cladding Swelling and Rupture Models for LOCA Analysis, NUREG-0630, April 1980.

[23] J. V. Cathcart et al., Zirconium Metal-Water Oxidation Kinetics IV. Reaction Rate Studies, ORNL/NUREG-17, August 1977.

[24] STAR-CD VERSION 4.06 Manual, CD-adapco, 2008.

[25] BWR (boiling Water Reactor) Refill-Reflood program Task 4.8 - Model Qualification Task plan, GE Co., San Jose, CA, NUREG/CR-1895, EPRI NP-1527, GEAP-24898, Aug., 1981.

[26] A. W. Bennett et al., Heat Transfer to Steam-Water Mixtures Flowing in Uniformly Heated Tubes in Which the Critical Heat Flux has been Exceeded, AERE-R5373, October 1976.

[27] E.-L. Pelletier, L.K.H. Leung, A. Teyssedou, and R. Girard, "Comparison and Improvements of Correlations for Film Boiling in Tubes," Proceedings of the 17th International Conference on Nuclear Engineering (ICONE17), July 1216, 2008, Brussels, Belgium.

[28] "SPACE code manual: Models and Correlations", KAERI, March 8, 2010

[29] Y. H. Kim, et al., V\&V Matrix for Safety Analysis Code SPACE, S06NX08-E-1-RD-08, Korea Electric Power Corporation Research Institute, 2009. 